\title{
Normal Quintic Surfaces which are Birationally Enriques Surfaces
}

\author{
By \\ Yumiko UMEZU*
}

\section{§0. Introduction}

Let $S$ be an Enriques surface over an algebraically closed field $k$ of characteristic $\neq 2$. Then, equivalently, $S$ is a non-singular projective surface with $q(S)=p_{g}(S)=0$ and $2 K_{S} \sim 0$. It is known (cf. Cossec [Co]) that every Enriques surface admits a morphism of degree one onto a surface of degree 10 in $\mathbf{P}^{5}$ with isolated rational double points, and also that every Enriques surface is birationally equivalent to a (non-normal) sextic surface in $\mathbf{P}^{3}$. Then there arises the following problem:

Problem. Can we birationally embed $S$ in $\mathbf{P}^{3}$ as a normal hypersurface? If yes, give the lower bound of the degree of the image.

If $X$ is a normal hypersurface of degree $d$ in $\mathbf{P}^{3}$, then $\omega_{X} \cong \mathcal{O}_{X}(d-4)$. Hence, if $\pi: \tilde{X} \rightarrow X$ is the minimal resolution of $X$, then $\omega_{\tilde{X}} \cong \pi^{*} \mathcal{C}_{\tilde{X}}(d-4) \otimes \mathcal{O}_{\tilde{X}}(-\tilde{D})$, where $\tilde{D}$ is an effective divisor on $\tilde{X}$, whose support coincides with the sum of the exceptional sets for non-rational singularities of $X$. So, for $X$ to be birationally an Enriques surface, it is necessary that $d \geq 5$ and $\tilde{D} \neq 0$. Castelnuovo [Ca] and Stagnaro [S] found normal quintic surfaces which are birationally equivalent to Enriques surfaces. In this paper we show (Theorem 2.1) that Enriques surfaces with certain conditions on their elliptic fibrations are birationally equivalent to normal quintic surfaces. Moreover we show (cf. Corollary 2.3) that generic Enriques surfaces and also all Enriques surfaces which are known to us now satisfy these conditions, and, conjecturally, so

Communicated by K. Saito, June 12, 1996. Revised December 17, 1996.

1991 Mathematics Subject Classifications: 14J28, 14E05, $14 \mathrm{~J} 17$.

* Department of Mathematics, School of Medicine, Toho University, 5-21-16, Omori-Nishi, Ota-ku, Tokyo 143, Japan. 
does every Enriques surface. $\S 3$ is devoted to proving Theorem 2.1 by constructing birational maps concretely. In $\$ 4$, we will study the singularity of the normal quintic surfaces constructed in $\$ 3$, and find their defining equations. It turns out that our quintic surfaces are those in Stagnaro [S]. Then we will prove, under a milder assumption than his, that the surfaces defined by the equations of this type are birationally equivalent to Enriques surfaces and are obtained by the construction of $\S 3$ (Theorem 4.3). Consequently the unirationality of the moduli space of Enriques surfaces is shown explicitly (cf. Cossec-Dolgachev [Co-D2] and Kondō [Ko]). In §1, we prove some properties of normal quintic surfaces, which are birationally Enriques surfaces. The author has found the construction in $\S 3$ from these observations.

For terminology and results on Enriques surfaces, we refer the reader to Barth-Peters-Van de Ven [B-P-V] and Cossec-Dolgachev [Co-D2]. For example, we call an effective divisor $E$ on an Enriques surface $S$ a halfpencil if $|2 E|$ is base point free and defines an elliptic fibration on $S$. Then there exists on $S$ a unique halfpencil $E^{\prime}$ adjoint with $E: E^{\prime} \sim E+K_{S}$. Note that a halfpencil is reduced, and is either a non-singular elliptic curve, a rational curve with one node, or a cycle of non-singular rational curves. For an irreducible curve $C, g(C)$ stands for the genus of the normalization of $C$, whereas $p_{a}(C)$ the arithmetic genus of $C$. If $Y_{1}$ and $Y_{2}$ are cycles on a variety, we shall denote their intersection by $Y_{1} Y_{2}$ or $Y_{1} . Y_{2}$. However, if it represents a 0 -cycle, then the intersection number of $Y_{1}$ and $Y_{2}$ is also denoted by $Y_{1} Y_{2}$.

After writing up the first version of this paper, the author received Yonggu Kim's paper [Ki], in which he claims that every Enriques surface is birationally equivalent to a normal quintic surface. But actually his argument is incomplete in proving the existence of a divisor which defines the birational map.

The author would like to express her thanks to Professor I.V. Dolgachev for discussions and informing her of the work of Kim and Castelnuovo.

The main results of this paper were announced in [U].

\section{$\S 1$. Birational Maps between Enriques Surfaces and Normal Quintic Surfaces}

Let $X$ be a normal quintic surface in $\mathbf{P}^{3}$. Assume that $X$ is birationally equivalent to an Enriques surface $S$. In this section, we study the birational map between $X$ and $S$.

Let $\pi: \tilde{S} \rightarrow X$ be the minimal resolution of all singularities on $X$. 
Proposition 1.1. $\operatorname{dim} R^{1} \pi_{*} \mathbb{C}_{\tilde{s}}=4$.

Proof. Consider the exact sequence:

$$
0 \rightarrow H^{1}\left(X, \mathcal{O}_{X}\right) \rightarrow H^{1}\left(\tilde{S}, \mathcal{O}_{\tilde{S}}\right) \rightarrow R^{1} \pi_{*} \mathcal{O}_{\tilde{S}} \rightarrow H^{2}\left(X, \mathcal{O}_{X}\right) \rightarrow H^{2}\left(\tilde{S}, \mathcal{O}_{\tilde{S}}\right) .
$$

Since $X$ is a quintic surface, $\operatorname{dim} H^{2}\left(X, \mathcal{O}_{X}\right)=\operatorname{dim} H^{0}\left(X, \mathcal{O}_{X}(1)\right)=4$. Since $\tilde{S}$ is birationally an Enriques surface, $H^{1}\left(\tilde{S}, \mathcal{O}_{\tilde{S}}\right)=0$ and $H^{2}\left(\tilde{S}, \mathcal{O}_{\tilde{S}}\right)=0$. Hence $\operatorname{dim} R^{1} \pi_{*} \mathcal{O}_{\tilde{s}}=4$.

Let $H \subset X$ be a general hyperplane section of $X$ and set $\tilde{H}=\pi^{*} H$. Then $H$ is a non-singular curve of genus 6 and $\tilde{H} \cong H$.

Proposition 1.2. $\operatorname{dim} H^{0}\left(\tilde{S}, \mathcal{O}_{\tilde{S}}(\tilde{H})\right)=4$, i.e. $\pi$ is defined by the complete linear system $|\tilde{H}|$.

Proof. From the exact sequence:

$$
0 \rightarrow \mathcal{O}_{\tilde{S}} \rightarrow \mathcal{O}_{\tilde{S}}(\tilde{H}) \rightarrow \mathcal{O}_{\tilde{H}}(\tilde{H}) \rightarrow 0,
$$

we have the long exact sequence:

$$
0 \rightarrow H^{0}\left(\tilde{S}, \mathcal{O}_{\tilde{S}}\right) \rightarrow H^{0}\left(\tilde{S}, \mathcal{O}_{\tilde{S}}(\tilde{H})\right) \rightarrow H^{0}\left(\tilde{H}, \mathcal{O}_{\tilde{H}}(\tilde{H})\right) \rightarrow H^{1}\left(\tilde{S}, \mathcal{C}_{\tilde{S}}\right) \rightarrow \cdots
$$

Since $H^{1}\left(\tilde{S}, \mathcal{O}_{\tilde{\mathbf{S}}}\right)=0$, we obtain

$$
\operatorname{dim} H^{0}\left(\tilde{S}, \mathcal{O}_{\tilde{S}}(\tilde{H})\right)=\operatorname{dim} H^{0}\left(\tilde{H}, \mathcal{O}_{\tilde{H}}(\tilde{H})\right)+1 .
$$

The Riemann-Roch Theorem for $\tilde{H}$ says that

$$
\operatorname{dim} H^{0}\left(\tilde{H}, \mathcal{O}_{\tilde{H}}(\tilde{H})\right)-\operatorname{dim} H^{1}\left(\tilde{H}, \mathcal{O}_{\tilde{H}}(\tilde{H})\right)=\tilde{H}^{2}-6+1=0 .
$$

Therefore, by Clifford's Theorem,

$$
\operatorname{dim} H^{0}\left(\tilde{H}, \mathcal{O}_{\tilde{H}}(\tilde{H})\right) \leq 3 .
$$

Hence $\operatorname{dim} H^{0}\left(\tilde{S}, \mathcal{O}_{\tilde{S}}(\tilde{H})\right) \leq 4$. Here the equality holds since $X$ is not contained in any hyperplane in $\mathbf{P}^{3}$.

Let $\mu: \tilde{S} \rightarrow S$ be the birational morphism from $\tilde{S}$ to $S$. Put $n=-K_{\tilde{S}}^{2}$. Then $\mu$ consists of $n$ blowing-downs:

$$
\tilde{S}=S_{0} \stackrel{\mu_{1}}{\rightarrow} S_{1} \stackrel{\mu_{2}}{\rightarrow} S_{2} \rightarrow \cdots \stackrel{\mu_{n}}{\rightarrow} S_{n}=S .
$$


There exists on $\tilde{S}$ an effective divisor $\tilde{D}$ such that $\tilde{H}-\tilde{D} \sim K_{\tilde{S}}$. $\quad \tilde{D}$ is supported by the sum of the exceptional sets of $\pi$, which correspond to non-rational singularities on $X$. Set $H_{i}=\left(\mu_{i} \circ \cdots \circ \mu_{1}\right)_{*}(\tilde{H}), D_{i}=\left(\mu_{i} \circ \cdots \circ \mu_{1}\right)_{*}(\tilde{D})$. We have $K_{S_{1}} \sim H_{i}-D_{i}$. Let $P_{i} \in S_{i}$ be the center of the blowing-up $\mu_{i}$, and let $A_{i}=\mu_{i}^{-1}\left(P_{i}\right)$. Moreover we denote by $\tilde{D}_{n}$ and $\tilde{A}_{i}$ the proper transform of $D_{n}$ and $A_{i}$ to $\tilde{S}$ respectively. Set $m_{i}=$ mult $_{P_{i}} H_{i}$ and $d_{i}=$ mult $_{P_{i}} D_{i}$.

Proposition 1.3. (i) $\tilde{D}^{2}=-5-n$.

(ii) $1 \leq n \leq 5$.

(iii) $m_{i}>0$ and $\sum_{i=1}^{n} m_{i}=5$.

(iv) $d_{i}>m_{i}$ and $\tilde{D}=\tilde{D}_{n}+\sum_{i=1}^{n}\left(d_{i}-m_{i}-1\right) \tilde{A}_{i}$.

Proof. (i) follows from $-n=K_{S}^{2}=\tilde{H}^{2}+\tilde{D}^{2}=5+\tilde{D}^{2}$. Let $A_{i}^{*}$ be the total transform of $A_{i}$ to $\tilde{S}$. Then we have first

$$
m_{i}=H_{i-1} A_{i}=\tilde{H} A_{i}^{*} .
$$

Since $\pi$ is the minimal resolution of $X$ and $A_{i}^{*}$ has at least one (-1)-curve as a component, $\tilde{H} A_{i}^{*}>0$ for every $i$. So $m_{i}>0$. Second, from

$$
K_{\tilde{S}} \sim \mu^{*} K_{S}+\sum_{i=1}^{n} A_{i}^{*} \equiv \sum_{i=1}^{n} A_{i}^{*},
$$

we have

$$
5=\tilde{H}^{2}=\tilde{H} K_{\tilde{S}}=\sum_{i=1}^{n} \tilde{H} A_{i}^{*},
$$

and hence (ii) and (iii) are proved. For (iv), let $e_{i}$ be the integers such that $\tilde{D}=\tilde{D}_{n}+\Sigma_{i=1}^{n} e_{i} \tilde{A}_{i}$. Then

$$
D_{i-1}=\mu_{i}^{*} D_{i}-d_{i} A_{i}+e_{i} A_{i} .
$$

Hence, with $H_{i-1}=\mu_{i}^{*} H_{i}-m_{i} A_{i}$, we have

$$
K_{S_{1-1}} \sim H_{i-1}-D_{i-1} \sim \mu_{i}^{*}\left(H_{i}-D_{i}\right)+\left(d_{i}-e_{i}-m_{i}\right) A_{i} \sim \mu_{i}^{*} K_{S_{t}}+\left(d_{i}-e_{i}-m_{i}\right) A_{i} .
$$

Thus we obtain $d_{i}-e_{i}-m_{i}=1$, and so $e_{i}=d_{i}-m_{i}-1$. Since $e_{i} \geq 0, d_{i} \geq m_{i}+1$. 


\section{§2. A Sufficient Condition for an Enriques Surface to be Birationally a Normal Quintic Surface}

Theorem 2.1. Let $S$ be an Enriques surface. Suppose there exist on $S$ three halfpencils $E_{1}, E_{2}, E_{3}$ such that:

(i) $E_{1} E_{2}=E_{2} E_{3}=E_{3} E_{1}=1$;

(ii) $\left(E_{1}+E_{1}^{\prime}\right) \cap E_{2} \cap E_{3}=\emptyset$, where $E_{1}^{\prime}$ denotes the halfpencil adjoint with $E_{1}$. Then $S$ is birationally equivalent to a normal quintic surface in $\mathbf{P}^{3}$.

We note, as we will see below, that there is no known example of an Enriques surface which does not satisfy the hypothesis in Theorem 2.1. The proof of Theorem 2.1 is given in the next section.

Cossec and Dolgachev [Co-D2] defined the non-degeneracy invariant $d(S)$ of an Enriques surface $S$, which is reformulated as follows:

$$
d(S)=\max \left\{\begin{array}{l}
r \\
\begin{array}{l}
\text { There exists on } S \text { halfpencils } E_{1}, \ldots, E_{r} \\
\text { such that } E_{i} E_{j}=1(1 \leq i<j \leq r)
\end{array}
\end{array}\right\} .
$$

Obviously the divisors $E_{1}, \ldots, E_{\mathrm{r}}$ as above are numerically independent, hence $d(S) \leq 10$. Cossec [Co] showed that $d(S)=10$ if $S$ contains no (-2)-curve, which happens, for example, if $S$ is generic (Barth-Peters [B-P], Cossec-Dolgachev [Co-D1]). On the other hand Cossec [Co] proved that $d(S) \geq 3$ for any Enriques surface $S$. But according to Cossec and Dolgachev [Co-D2], no Enriques surface with $d(S)=3$ is known.

As for the condition (ii) of the Theorem 2.1, we have the following:

Proposition 2.2. Every Enriques surface $S$ with $d(S) \geq 4$ admits halfpencils $E_{1}, E_{2}, E_{3}$ satisfying the hypothesis (i) and (ii) of Theorem 2.1.

Hence we obtain:

Corollary 2.3. Every Enriques surface $S$ with $d(S) \geq 4$ is birationally equivalent to a normal quintic surface in $\mathbf{P}^{3}$.

To prove Proposition 2.2, we will begin with the following

Lemma 2.4. Let $E_{1}, E_{2}, E_{3}$ be halfpencils on an Enriques surface $S$ such that $E_{1} E_{2}=E_{2} E_{3}=E_{3} E_{1}=1$. Let $E_{i}^{\prime}$ denote the halfpencil adjoint with $E_{i}(i=1,2,3)$. 
If $E_{1}, E_{2}$ and $E_{3}$ meet at one common point, then so do $E_{i}, E_{j}^{\prime}$ and $E_{k}^{\prime}$, $\{i, j, k\}=\{1,2,3\}$.

Proof. First we note that $E_{i}$ and $E_{j}(i \neq j)$ have no common components. (This can be seen easier in our situation, where $E_{i}$ is at most a cycle of reduced (-2)-curves. A proof for a general situation is given in the Appendix.) Then we can define four different points $P_{1}=E_{1} \cap E_{2}, P_{2}=E_{1} \cap E_{2}^{\prime}, P_{3}=E_{1}^{\prime} \cap E_{2}$, $P_{4}=E_{1}^{\prime} \cap E_{2}^{\prime}$. By assumption, $P_{1} \in E_{3}$. Then, since $\mathcal{O}_{E_{1}}\left(E_{2}-E_{2}^{\prime}\right) \cong \mathcal{O}_{E_{1}}\left(E_{3}-E_{3}^{\prime}\right)$ and $p_{a}\left(E_{1}\right)=1$, we have $E_{1} \cap E_{3}^{\prime}=P_{2}$. Similarly $E_{2} \cap E_{3}^{\prime}=P_{3}$ by $\mathcal{O}_{E_{2}}\left(E_{1}-E_{1}^{\prime}\right)$ $\cong \mathcal{O}_{E_{2}}\left(E_{3}-E_{3}^{\prime}\right)$ and $p_{a}\left(E_{2}\right)=1$. Hence, by $\mathcal{O}_{E_{1}^{\prime}}\left(E_{2}-E_{2}^{\prime}\right) \cong \mathcal{O}_{E_{1}^{\prime}}\left(E_{3}^{\prime}-E_{3}\right)$ and $p_{a}\left(E_{1}^{\prime}\right)=1$, we have $E_{1}^{\prime} \cap E_{3}=P_{4}$.

Proof of Proposition 2.2. Let $E_{1}, E_{2}, E_{3}, E_{4}$ be any halfpencils on $S$ with $E_{i} E_{j}=1(1 \leq i \neq j \leq 4)$. Let $E_{i}^{\prime}$ denote the halfpencil adjoint with $E_{i}(1 \leq i \leq 4)$. Let $P_{1}, \ldots, P_{4}$ be the same as in the proof of Lemma 2.4. If $E_{3}$ [resp. $\left.E_{4}\right]$ passes through neither $P_{1}$ nor $P_{3}$, then $E_{1}, E_{2}, E_{3}$ [resp. $E_{1}, E_{2}, E_{4}$ ] satisfy the conditions (i) and (ii) of Theorem 2.1.

Let us suppose that $E_{3}$ and $E_{4}$ pass through either $P_{1}$ or $P_{3}$. Then, by Lemma 2.4, we can assume that $P_{1}, P_{4} \in E_{3}, E_{4}$, by exchanging $E_{3}$ for $E_{3}^{\prime}$ or $E_{4}$ for $E_{4}^{\prime}$ if necessary. Recall that $E_{3}$ and $E_{4}$ have no common components. Then we obtain $E_{3} E_{4} \geq 2$, which contradicts our hypothesis.

\section{§3. Construction of Birational Maps}

In this section we prove Theorem 2.1. For that, we shall explicitly

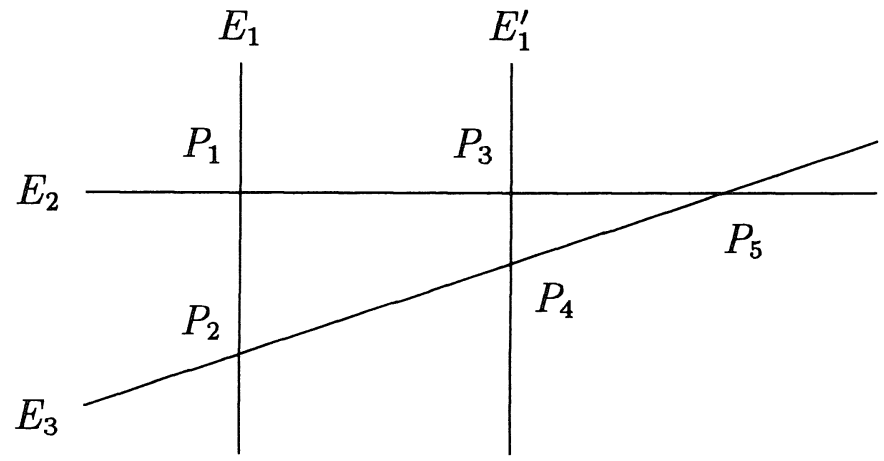

Figure 1 
construct birational maps from Enriques surfaces, which satisfy the hypothesis of the Theorem, to normal quintic surfaces.

Let $S, E_{1}, E_{1}^{\prime}, E_{2}, E_{3}$ be as in Theorem 2.1. Set $\bar{D}=E_{1}+E_{1}^{\prime}+E_{2}+E_{3}$ and $P_{1}=E_{1} \cap E_{2}, P_{2}=E_{1} \cap E_{3}, P_{3}=E_{1}^{\prime} \cap E_{2}, P_{4}=E_{1}^{\prime} \cap E_{3}, P_{5}=E_{2} \cap E_{3}$, so that $P_{1}, \ldots, P_{5}$ are the set of double points of $\bar{D}$ other than singular points of $E_{1}$, $E_{1}^{\prime}, E_{2}$ and $E_{3}$ (see Figure 1).

Let $\bar{H}$ be a general member of $\left|\bar{D}+K_{S}\right|=\left|2 E_{1}+E_{2}+E_{3}\right|$.

Lemma 3.1. (i) $\bar{H}^{2}=10$.

(ii) $\operatorname{dim} H^{0}\left(S, \mathcal{O}_{S}(\bar{H})\right)=6$.

Proof. (i) is clear since $E_{i} E_{j}=1-\delta_{i j}(1 \leq i, j \leq 3)$. For (ii), we have by the Riemann-Roch Theorem and (i),

$$
\begin{aligned}
\operatorname{dim} & H^{0}\left(S, \mathcal{O}_{S}(\bar{H})\right)-\operatorname{dim} H^{1}\left(S, \mathcal{O}_{S}(\bar{H})\right)+\operatorname{dim} H^{2}\left(S, \mathcal{O}_{S}(\bar{H})\right) \\
= & \frac{1}{2} \bar{H}\left(\bar{H}-K_{S}\right)+1=6 .
\end{aligned}
$$

$H^{2}\left(S, \mathcal{O}_{S}(\bar{H})\right)$ is the dual space of $H^{0}\left(S, \mathcal{O}_{S}\left(K_{S}-\bar{H}\right)\right)=H^{0}\left(S, \mathcal{O}_{S}(-\bar{D})\right)=0$, and $H^{1}\left(S, \mathcal{O}_{S}(\bar{H})\right)$ is that of $H^{1}\left(S, \mathcal{O}_{S}(-\bar{D})\right)$. We will prove that $H^{1}\left(S, \mathcal{O}_{S}(-\bar{D})\right)=0$. Considering the exact sequence

$$
\begin{aligned}
0 & \rightarrow H^{0}\left(S, \mathcal{O}_{S}(-\bar{D})\right) \rightarrow H^{0}\left(S, \mathcal{O}_{S}\right) \rightarrow H^{0}\left(\bar{D}, \mathcal{O}_{\bar{D}}\right) \\
& \rightarrow H^{1}\left(S, \mathcal{O}_{s}(-\bar{D})\right) \rightarrow H^{1}\left(S, \mathcal{O}_{S}\right) \rightarrow \cdots
\end{aligned}
$$

it is enough to show $H^{0}\left(-D, C_{\bar{D}}\right) \cong k$. In fact, since $\bar{D}$ is a union of different halfpencils, $\bar{D}$ is connected and reduced. Hence $H^{0}\left(\bar{D}, \mathcal{O}_{\bar{D}}\right) \cong k$.

For $i=2,3$, let $E_{i}^{\prime}$ denote the halfpencil adjoint with $E_{i}$. By Lemma 2.4 and our assumption on $E_{1}, E_{1}^{\prime}, E_{2}, E_{3}$, we see that $E_{2}^{\prime}$ and $E_{3}^{\prime}$ do not pass through any of $P_{1}, \ldots, P_{5}$.

Lemma 3.2. Let $s_{0}, \ldots, s_{5}$ be sections of $H^{0}\left(S, \mathcal{O}_{S}(\bar{H})\right)$ such that $\left(s_{0}\right)_{0}=2 E_{1}^{\prime}+E_{2}+E_{3},\left(s_{1}\right)_{0}=2 E_{1}+E_{2}+E_{3},\left(s_{2}\right)_{0}=E_{1}+E_{1}^{\prime}+E_{2}^{\prime}+E_{3},\left(s_{3}\right)_{0}=E_{1}$ $+E_{1}^{\prime}+E_{2}+E_{3}^{\prime},\left(s_{4}\right)_{0}=2 E_{1}+E_{2}^{\prime}+E_{3}^{\prime},\left(s_{5}\right)_{0}=2 E_{1}^{\prime}+E_{2}^{\prime}+E_{3}^{\prime}$. Then $s_{0}, \ldots, s_{5}$ form a basis of $H^{0}\left(S, \mathcal{O}_{S}(\bar{H})\right)$.

Proof. By the definition of $E_{i}$ and $E_{i}^{\prime}$, it is easy to check that $s_{0}, \ldots, s_{5}$ 
belong to $H^{0}\left(S, \mathcal{O}_{S}(\bar{H})\right)$. From Lemma 3.1 (ii), it suffices to prove that $s_{0}, \ldots, s_{5}$ are linearly independent. Put $\bar{H}_{i}=\left(s_{i}\right)_{0} \quad(0 \leq i \leq 5)$. Then $\bar{H}_{0}$ and $\bar{H}_{1}$ have $E_{2}+E_{3}$ as fixed components, but $\bar{H}_{2}$ does not; $\bar{H}_{0}, \bar{H}_{1}$ and $\bar{H}_{2}$ have $E_{3}$ as a fixed component, but $\bar{H}_{3}$ does not; $\bar{H}_{0}, \ldots, \bar{H}_{3}$ have $P_{1}, \ldots, P_{5}$ as base points, but $\bar{H}_{4}$ does not pass through $P_{3}, P_{4}, P_{5} ; \bar{H}_{0}, \ldots, \bar{H}_{4}$ have $P_{1}$ and $P_{2}$ as base points, but $\bar{H}_{5}$ does not pass through neither. Therefore $s_{0}, \ldots, s_{5}$ are linearly independent.

Let $\mu: \tilde{S} \rightarrow S$ be the blowing-up at $P_{1}, \ldots, P_{5}$ and $\tilde{l}_{i}$ the $(-1)$-curve over $P_{i}(1 \leq i \leq 5)$. Let $\tilde{H}$ be the proper transform of a general member of the linear subspace $\Lambda=\left|\bar{H}-P_{1}-\cdots-P_{5}\right|$ of $|\bar{H}|$.

Corollary 3.3. (i) $\operatorname{dim} H^{0}\left(\tilde{S}, \mathcal{O}_{\tilde{S}}(\tilde{H})\right)=4$.

(ii) $\tilde{H} \in\left|\mu^{*}(\bar{H})-\tilde{l}_{i}-\cdots-\tilde{l}_{5}\right|$.

(iii) $\tilde{H}^{2}=5$.

(iv) $\mathrm{Bs}|\tilde{H}|=\emptyset$.

(v) $\tilde{H}$ is an irreducible curve with $p_{a}(\tilde{H})=6$.

Proof. (i): In the proof of Lemma 3.2, we saw that $s_{0}, \ldots, s_{3}$ form homogeneous coordinates of the space $\Lambda$, and hence $4=\operatorname{dim} \Lambda+1=\operatorname{dim} H^{0}(\tilde{S}$, $\mathcal{O}_{\tilde{S}}(\tilde{H})$ ). Moreover we have that $\operatorname{Bs} \Lambda=\left\{P_{1}, \ldots, P_{5}\right\}$.

Proving (ii), (iii) and (iv) is then equivalent to showing, by Lemma 3.1 (i), that for each $P_{i}$ there exist $i_{1}$ and $i_{2}\left(0 \leq i_{1} \neq i_{2} \leq 3\right)$ such that $\bar{H}_{i_{1}}$ and $\bar{H}_{i_{2}}$ are smooth at $P_{i}$ and have different tangents at $P_{i}$, where $\bar{H}_{j}=\left(s_{j}\right)_{0}$. Indeed we find such $\bar{H}_{i_{1}}$ and $\bar{H}_{i_{2}}$ as follows:

$$
\begin{array}{ll}
P_{1}: & \bar{H}_{0}, \bar{H}_{2} \\
P_{2}: & \bar{H}_{0}, \bar{H}_{3} \\
P_{3}: & \bar{H}_{1}, \bar{H}_{2} \\
P_{4}: & \bar{H}_{1}, \bar{H}_{3} \\
P_{5}: & \bar{H}_{2}, \bar{H}_{3} .
\end{array}
$$

(v): By (iv), $\tilde{H}$ is irreducible. Moreover we have proved that $\mu_{*} \tilde{H}$ is also non-singular at $P_{1}, \ldots, P_{5}$. Hence $p_{a}(\tilde{H})=p_{a}(\bar{H})=1 / 2 \bar{H}^{2}+1=6$ by Lemma 3.1, (i).

This Corollary says that $|\tilde{H}|$ defines a morphism from $\tilde{S}$ to $\mathbb{P}^{3}$ and its image 
is a surface. Let $X$ denote the image and $\pi: \tilde{S} \rightarrow X$ the induced morphism.

Lemma 3.4. $\pi$ is a birational morphism and $\operatorname{deg} X=5$.

Proof. Let $H$ be a general hyperplane section of $X \subset \mathbf{P}^{3}$. Then we may assume $\tilde{H}=\pi^{*} H$, and hence $\tilde{H}^{2}=\operatorname{deg} \pi \cdot H^{2}$. By Corollary 3.3 (iii), we obtain $\operatorname{deg} \pi=1$ and $H^{2}=5$.

\section{Lemma 3.5. $X$ is normal.}

Proof. The restriction morphism $\pi_{\mid \tilde{H}}: \tilde{H} \rightarrow H$ is a birational morphism onto a plane quintic curve $H$. From Corollary 3.3 , (v), we get $p_{a}(\tilde{H})=6=p_{a}(H)$, which implies that the surface $X$ has only isolated singularities, and hence that $X$ is normal.

Therefore $\pi \circ \mu^{-1}$ is a birational map from $S$ to a normal quintic surface $X$, and hence Theorem 2.1 is proved.

\section{$\S 4$. Singularity of $X$ and Defining Equations}

Let $S$ be an Enriques surface which satisfies the hypothesis of Theorem 2.1 , and $X$ the quintic surface birationally equivalent to $S$ as constructed from $S$ in $\S 3$. We use also the other notations such as $E_{i}, E_{i}^{\prime}, \bar{H}, \bar{D}, s_{i}, \mu, \tilde{H}, \tilde{l}_{i}$, $\pi$ as in $\S 2$ and $\S 3$.

Let $\tilde{E}_{i}$ and $\tilde{E}_{i}^{\prime}$ be the proper transform of $E_{i}$ and $E_{i}^{\prime}$ to $\tilde{S}$ respectively $(1 \leq i \leq 3)$. Then $\tilde{E}_{1}, \tilde{E}_{1}^{\prime}, \tilde{E}_{2}, \tilde{E}_{3}$ are disjoint from each other, $\widetilde{E}_{1}^{2}=\tilde{E}_{1}^{\prime 2}=-2$ and $\widetilde{E}_{2}^{2}=\widetilde{E}_{3}^{2}=-3$. On $E_{i}$ and $E_{i}^{\prime}, \mu$ is at most blowing-up at non-singular points for any $i$. Hence $p_{a}\left(\tilde{E}_{i}\right)=p_{a}\left(\tilde{E}_{i}^{\prime}\right)=1$. Moreover $p_{a}(A) \leq 0$ for any non-zero effective divisor $A$ such that $A<\tilde{E}_{i}$ or $A<\tilde{E}_{i}^{\prime}$, and $\tilde{E}_{i}$ and $\tilde{E}_{i}^{\prime}$ has no (-1)-curve as a component. Since $\tilde{E}_{1}, \tilde{E}_{1}^{\prime}, \tilde{E}_{2}, \tilde{E}_{3}$ are disjoint from $\tilde{H}$, the image on $X$ of these divisors are singular points.

Let $\bar{H}_{i}$ be as in the proof of Lemma 3.2. Set $\tilde{H}_{i}=\mu^{*} \bar{H}_{i}-\tilde{l}_{1}-\cdots-\tilde{l}_{5}$ $(0 \leq i \leq 3)$. Then $\tilde{H}_{i} \in|\tilde{H}|$ by Corollary 3.3 (ii), and

$$
\begin{aligned}
& \tilde{H}_{0}=2 \tilde{E}_{1}^{\prime}+\tilde{E}_{2}+\tilde{E}_{3}+2 \tilde{l}_{3}+2 \tilde{l}_{4}+\tilde{l}_{5} \\
& \tilde{H}_{1}=2 \tilde{E}_{1}+\tilde{E}_{2}+\tilde{E}_{3}+2 \tilde{l}_{1}+2 \tilde{l}_{2}+\tilde{l}_{5} \\
& \tilde{H}_{2}=\tilde{E}_{1}+\tilde{E}_{1}^{\prime}+\tilde{E}_{2}^{\prime}+\tilde{E}_{3}+\tilde{l}_{2}+\tilde{l}_{4}
\end{aligned}
$$




$$
\tilde{H}_{3}=\widetilde{E}_{1}+\tilde{E}_{1}^{\prime}+\tilde{E}_{2}+\tilde{E}_{3}^{\prime}+\tilde{l}_{1}+\tilde{l}_{3} .
$$

Since $\tilde{H} \tilde{l}_{i}=1$, the image of $\tilde{l}_{i}$ on $X$ is a line for $1 \leq i \leq 5$. Therefore we have first that $\widetilde{E}_{1}, \widetilde{E}_{1}^{\prime}, \widetilde{E}_{2}, \widetilde{E}_{3}$ are contracted by $\pi$ to distinct singular points, which we call $Q_{0}, Q_{1}, Q_{2}, Q_{3}$ respectively. We know (Laufer [L]) that each of them is either a simple elliptic or a cusp singularity. The other singularities, if they exist, are rational double points (cf. Proposition 1.1). Second, if $\tilde{C}$ is a curve on $\tilde{S}$ which is contracted by $\pi$ to a rational double point, then $\tilde{H}_{i} \tilde{C}=0$ for every $i$. Hence $\tilde{C}$ is disjoint from $\tilde{E}_{1}+\tilde{E}_{1}^{\prime}+\tilde{E}_{2}+\tilde{E}_{3}+\tilde{l}_{1}+\cdots+\tilde{l}_{5}$, and therefore $C=\mu_{*} \tilde{C}$ is disjoint from $\bar{D}$. Conversely, if an irreducible curve $C$ on $S$ is disjoint from $\bar{D}$, then $C$ is a $(-2)$-curve and is also disjoint from $\bar{H}$, and so $\tilde{C}=\mu^{*} C$ is a $(-2)$-curve, which is disjoint from $\tilde{H}$ and $\tilde{E}_{1}+\tilde{E}_{1}^{\prime}+\tilde{E}_{2}+\tilde{E}_{3}$. Hence the image of $\tilde{C}$ on $X$ is a rational double point.

For a singular point, let $Z$ be the fundamental cycle of its minimal resolution. Then to sum up we have

Proposition 4.1. The singularity of $X$ consists of

1) two singularities with $Z^{2}=-2$, each of which is either a simple elliptic or a cusp singularity; these correspond to $E_{1}$ and $E_{1}^{\prime}$ on $S$;

2) two singularities with $Z^{2}=-3$, each of which is either a simple elliptic or a cusp singularity; these correspond to $E_{2}$ and $E_{3}$ on $S$; and possibly

3) rational double points; each of them corresponds to a connected component of the sum of all curves on $S$, which are disjoint from $\bar{D}$. Moreover, $\tilde{S}$ is the minimal resolution of all singularities on $X$.

Now we are going to find the defining equation of $X$ in $\mathbb{P}^{3}$. Lemma 3.2 and Corollary 3.3 (ii) show that we can choose homogeneous coordinates $\left(X_{0}: X_{1}: X_{2}: X_{3}\right)$ of $\mathbf{P}^{3}$ so that $\pi^{*} H_{i}=\tilde{H}_{i}(0 \leq i \leq 3)$, where $H_{i}$ denotes the section of $X$ by the hyperplane $\left\{X_{i}=0\right\}$. We fix such coordinates. $\tilde{H}_{0}$ is disjoint from $\tilde{E}_{1}$, while $\tilde{H}_{i} \geq \tilde{E}_{1}$ for $i \neq 0$. Therefore $Q_{0}=(1: 0: 0: 0)$. Similarly we have $Q_{1}=(0: 1: 0: 0), Q_{2}=(0: 0: 1: 0)$ and $Q_{3}=(0: 0: 0: 1)$. By $\widetilde{E}_{1}^{2}=\widetilde{E}_{1}^{\prime 2}=-2, Q_{0}$ and $Q_{1}$ are double points; by $\tilde{E}_{2}^{2}=\widetilde{E}_{3}^{2}=-3, Q_{2}$ and $Q_{3}$ are triple points (Laufer [L]).

Let $l_{i}$ denote the line $\pi_{*} \tilde{l}_{i}(1 \leq i \leq 5)$. Then their defining equations are as follows (see Figure 2): 


$$
\begin{aligned}
& l_{1}: X_{1}=X_{3}=0 \\
& l_{2}: X_{1}=X_{2}=0 \\
& l_{3}: X_{0}=X_{3}=0 \\
& l_{4}: X_{0}=X_{2}=0 \\
& l_{5}: X_{0}=X_{1}=0
\end{aligned}
$$

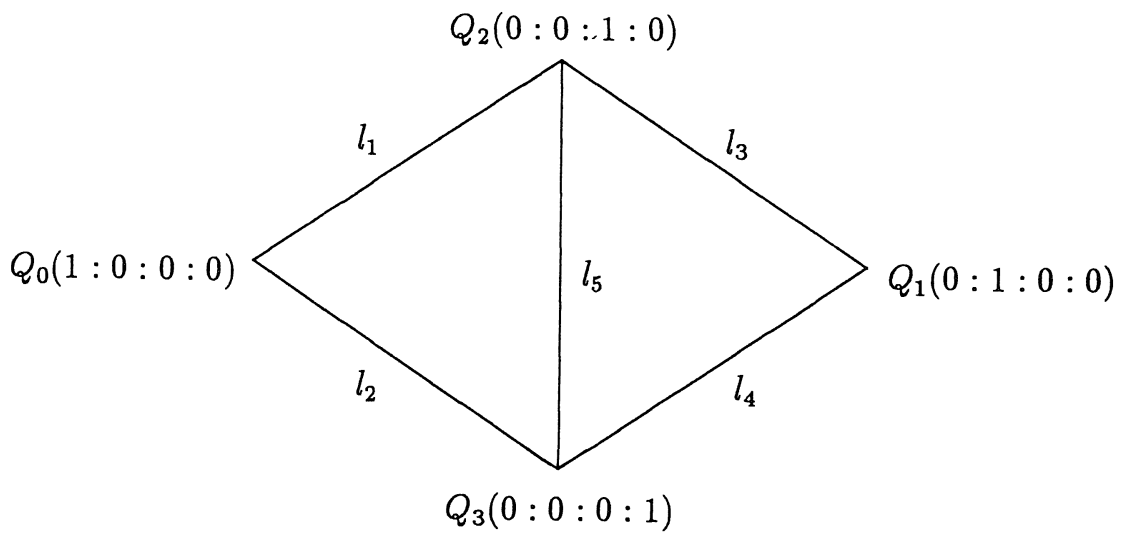

Figure 2

The defining equation of $X$ in $\mathbf{P}^{3}$ corresponds to a non-trivial linear equation relating the monomials $\left\{s_{0}^{n_{0}} s_{1}^{n_{1}} s_{2}^{n_{2}} s_{3}^{n_{3}} \mid n_{0}+n_{1}+n_{2}+n_{3}=5\right\}$ in $H^{0}\left(S, \mathcal{O}_{s}(5 \bar{H})\right)$. We find 14 out of these monomials, whose divisors of zeros are greater than $4 E_{1}+4 E_{1}^{\prime}+3 E_{2}+3 E_{3}$, as follows:

$$
\begin{gathered}
s_{0}^{3} s_{1}^{2}, s_{0}^{2} s_{1}^{3}, s_{0}^{2} s_{1} s_{2}^{2}, s_{0}^{2} s_{1} s_{3}^{2}, s_{0}^{2} s_{1}^{2} s_{2}, s_{0}^{2} s_{1}^{2} s_{3}, s_{0}^{2} s_{1} s_{2} s_{3}, \\
s_{0} s_{1}^{2} s_{2}^{2}, s_{0} s_{1}^{2} s_{3}^{2}, s_{0} s_{2}^{2} s_{3}^{2}, s_{0} s_{1}^{2} s_{2} s_{3}, s_{0} s_{1} s_{2}^{2} s_{3}, s_{0} s_{1} s_{2} s_{3}^{2}, s_{1} s_{2}^{2} s_{3}^{2} .
\end{gathered}
$$

We shall show that these are linearly dependent. Set $G=5 \bar{H}-\left(4 E_{1}+4 E_{1}^{\prime}\right.$ $\left.+3 E_{2}+3 E_{3}\right) \sim 2 E_{1}+2 E_{2}+2 E_{3}$ and $G^{\prime}=E_{1}+E_{1}^{\prime}+F_{2}+F_{3}$, where $F_{2}$ and $F_{3}$ are general members of $\left|2 E_{2}\right|$ and $\left|2 E_{3}\right|$ respectively. Then $G^{\prime}$ is connected and reduced, so $\operatorname{dim} H^{0}\left(G^{\prime}, \mathcal{O}_{G^{\prime}}\right)=1$. Moreover $G^{\prime} \sim G-K_{S}$. By the exact sequence

$$
\begin{aligned}
0 & \rightarrow H^{0}\left(S, \mathcal{O}_{S}\left(-G^{\prime}\right)\right) \rightarrow H^{0}\left(S, \mathcal{O}_{S}\right) \rightarrow H^{0}\left(G^{\prime}, \mathcal{O}_{G^{\prime}}\right) \\
& \rightarrow H^{1}\left(S, \mathcal{O}_{S}\left(-G^{\prime}\right)\right) \rightarrow H^{1}\left(S, \mathcal{O}_{S}\right) \rightarrow \cdots,
\end{aligned}
$$


we have $H^{1}\left(S, \mathcal{O}_{S}\left(-G^{\prime}\right)\right)=0$ and so $H^{1}\left(S, \mathcal{O}_{S}(G)\right)=0$. Therefore, with $H^{2}(S$, $\left.\mathcal{O}_{S}(G)\right) \cong H^{0}\left(S, \mathcal{O}_{S}\left(-G^{\prime}\right)\right)^{\vee}=0$, we obtain by the Riemann-Roch Theorem

$$
\begin{aligned}
\operatorname{dim} H^{0}\left(S, \mathcal{O}_{S}(G)\right) & =1 / 2 G\left(G-K_{S}\right)+1 \\
& =13 .
\end{aligned}
$$

Thus there exists a non-trivial linear relation among the 14 monomials above, and hence $X$ is defined by an equation of the form:

(*) $\quad F=a_{1} X_{0}^{3} X_{1}^{2}+a_{1} X_{0}^{2} X_{1}^{3}+a_{3} X_{0}^{2} X_{1} X_{2}^{2}+a_{4} X_{0}^{2} X_{1} X_{3}^{2}$

$$
\begin{aligned}
& +a_{5} X_{0}^{2} X_{1}^{2} X_{2}+a_{6} X_{0}^{2} X_{1}^{2} X_{3}+a_{7} X_{0}^{2} X_{1} X_{2} X_{3}+a_{8} X_{0} X_{1}^{2} X_{2}^{2} \\
& +a_{9} X_{0} X_{1}^{2} X_{3}^{2}+a_{10} X_{0} X_{2}^{2} X_{3}^{2}+a_{11} X_{0} X_{1}^{2} X_{2} X_{3} \\
& +a_{12} X_{0} X_{1} X_{2}^{2} X_{3}+a_{13} X_{0} X_{1} X_{2} X_{3}^{2}+a_{14} X_{1} X_{2}^{2} X_{3}^{2}
\end{aligned}
$$$$
=0
$$

$$
\left(a_{1}, \ldots, a_{14} \in k\right)
$$

Since $X$ has double points at $Q_{0}=(1: 0: 0: 0)$ and $Q_{1}=(0: 1: 0: 0), a_{1}, a_{2} \neq 0$. If $a_{10}=0$, then $X_{1} \mid F$; if $a_{14}=0$, then $X_{0} \mid F$. Hence $a_{10}, a_{14} \neq 0$. If $a_{3}=0$, then each monomial of $F$ is divisible by $X_{1}^{2}, X_{1} X_{3}$ or $X_{3}^{2}$, and so $X$ has singularity along $l_{1}$. Similarly $X$ has singularity along $l_{2}$ if $a_{4}=0$, along $l_{3}$ if $a_{8}=0$, along $l_{4}$ if $a_{9}=0$. Since $X$ is normal, we obtain $a_{3}, a_{4}, a_{8}, a_{9} \neq 0$.

Stagnaro [S] has given these equations as one type of examples of quintics, whose resolutions are (blowing-up of) Enriques surfaces. Here we study them with regard to our construction in $\S 3$.

By multiplying some coordinates $X_{i}$ by non-zero constants, we can assume, for example, $a_{1}=a_{2}=a_{3}=a_{4}=1$. Thus we have proved the following:

Theorem 4.2. Let $X$ be a normal quintic surface in $\mathbb{P}^{3}$, which is constructed in the way of $\$ 3$ from an Enriques surface $S$ satisfying the hypothesis of Theorem 2.1. Then, with suitable homogeneous coordinates $\left(X_{0}: X_{1}: X_{2}: X_{3}\right)$ of $\mathbb{P}^{3}$, the defining equation of $X$ is of the following form:

$$
\begin{gathered}
(* *) \quad F=X_{0}^{3} X_{1}^{2}+X_{0}^{2} X_{1}^{3}+X_{0}^{2} X_{1} X_{2}^{2}+X_{0}^{2} X_{1} X_{3}^{2} \\
+c_{1} X_{0}^{2} X_{1}^{2} X_{2}+c_{2} X_{0}^{2} X_{1}^{2} X_{3}+c_{3} X_{0}^{2} X_{1} X_{2} X_{3}+c_{4} X_{0} X_{1}^{2} X_{2}^{2} \\
+c_{5} X_{0} X_{1}^{2} X_{3}^{2}+c_{6} X_{0} X_{2}^{2} X_{3}^{2}+c_{7} X_{0} X_{1}^{2} X_{2} X_{3} \\
+c_{8} X_{0} X_{1} X_{2}^{2} X_{3}+c_{9} X_{0} X_{1} X_{2} X_{3}^{2}+c_{10} X_{1} X_{2}^{2} X_{3}^{2}
\end{gathered}
$$


$=0$

$$
\left(c_{4}, c_{5}, c_{6}, c_{10} \neq 0, c_{1}, \ldots, c_{10} \in k\right)
$$

Remark. The condition $c_{4}, c_{5}, c_{6}, c_{10} \neq 0$ in Theorem 4.2 is not a sufficient condition for $X=\{F=0\}$ to be a normal surface.

In what follows we shall prove the converse of our construction, namely:

Theorem 4.3. Let $F=0$ be the equation of the form (**). Set $X=\{F=0\} \subset \mathbf{P}^{3}$, and suppose that $X$ has at worst isolated rational singularities

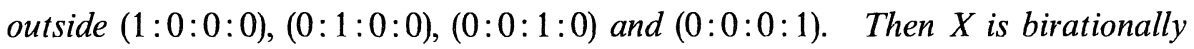
equivalent to an Enriques surface $S$, which satisfies the hypothesis in Theorem 2.1 , and $X$ is constructed from $S$ in the way described in $\S 3$.

Remark. Stagnaro [S] proved that the surface defined by the equation $(*)$ is birationally equivalent to an Enriques surface under the assumption that the singularity of the surface is the same as what may arise from an Enriques surface, which is the case, for example, if the coefficients $a_{i}$ 's are general.

Corollary 4.4. Let $M$ be the moduli space of the quadruple $\left(S, E_{1}, E_{2}, E_{3}\right)$ as in Theorem 2.1. Then $M$ is isomorphic to an open subset of the affine space $\mathbf{A}^{10}$. In particular, the moduli space of the Enriques surfaces is unirational.

Before proving the Theorem, we note the following elementary facts.

Lemma 4.5. Let $Y$ be a non-singular surface and $\sigma: \tilde{Y} \rightarrow Y$ the blowing-up at a point $P \in Y$. Let $\tilde{D}$ be an effective divisor on $\tilde{Y}$. Then we have $p_{a}(\tilde{D}) \leq p_{a}\left(\sigma_{*} \tilde{D}\right)$.

Proof. Let $E$ denote the exceptional curve for $\sigma$. Set $D=\sigma_{*} \tilde{D}$. Then $\tilde{D}=\sigma^{*} D+p E$ for some $p \in \mathbb{Z}$. If $p=0$, then

$$
\begin{aligned}
p_{a}(\tilde{D}) & =p_{a}\left(\sigma^{*} D\right)=1 / 2\left(\sigma^{*} D\right)\left(\sigma^{*} D+\sigma^{*} K_{Y}+E\right)+1 \\
& =1 / 2 D\left(D+K_{Y}\right)+1=p_{a}(D) .
\end{aligned}
$$

For $m>0$, we have 


$$
p_{a}(m E)=1 / 2(m E)\left(m E+\sigma^{*} K_{Y}+E\right)+1=1-1 / 2 m(m+1) \leq 0 .
$$

Hence if $p>0$, then

$$
p_{a}(\tilde{D})=p_{a}\left(\sigma^{*} D\right)+p_{a}(p E)+\left(\sigma^{*} D\right)(p E)-1<p_{a}\left(\sigma^{*} D\right)=p_{a}(D) .
$$

If $p<0$, put $m=-p$. Then

$$
\begin{aligned}
p_{a}(D) & =p_{a}\left(\sigma^{*} D\right)=p_{a}\left(\sigma^{*} D-m E\right)+p_{a}(m E)+\left(\sigma^{*} D-m E\right)(m E)-1 \\
& =p_{a}(\tilde{D})+1-1 / 2 m(m+1)+m^{2}-1 \geq p_{a}(\tilde{D}) .
\end{aligned}
$$

Corollary 4.6. Let $\sigma: \tilde{Y} \rightarrow Y$ be a birational morphism between non-singular surfaces. Let $\tilde{A}$ be a curve on $\tilde{Y}$ and set $A=\sigma(\tilde{A})$. Then

$$
\sup _{\substack{\tilde{D}>0 \\ \text { Supp } \tilde{D} \subseteq \tilde{A}}} p_{a}(\tilde{D}) \leq \sup _{\substack{D>0 \\ \operatorname{Supp} D \subseteq A}} p_{a}(D) .
$$

Proof. This follows from Lemma 4.5, since $\sigma$ consists of blowing-ups.

Proof of Theorem 4.3. The original form $(*)$ of $F$ with the condition $a_{1}, a_{2}, a_{3}, a_{4}, a_{8}, a_{9}, a_{10}, a_{14} \neq 0$ is symmetric with respect to $X_{0}$ and $X_{1}$; and $X_{2}$ and $X_{3}$. Hence $X=\{F=0\}$ has the same symmetry property, unless we assume some additional condition, which is incompatible with it. The reduction procedure from $(*)$ to $(* *)$ and the assumptions on the singularity of $X$ in our Theorem are compatible with this symmetry.

Set $Q_{0}=(1: 0: 0: 0), Q_{1}=(0: 1: 0: 0), Q_{2}=(0: 0: 1: 0)$ and $Q_{3}=(0: 0: 0: 1)$. Then $Q_{0}$ and $Q_{1}$ are double points, and $Q_{2}$ and $Q_{3}$ are triple points. Let $\pi: \tilde{S} \rightarrow X$ be the minimal resolution of $X$ and $\tilde{H}=\pi^{*} H$, where $H$ is a hyperplane section of $X$. Then there exists an effective divisor $\tilde{D}$ on $\tilde{S}$ such that (1) $\operatorname{Supp} \tilde{D}$ is included in the union of the exceptional sets over $Q_{0}, Q_{1}, Q_{2}$ and $Q_{3}$, and (2) $K_{\tilde{S}} \sim \tilde{H}-\tilde{D}$. Let $H_{0}, H_{1}, H_{2}$ denote the hyperplane sections of $X$ through $Q_{0}, Q_{2}, Q_{3} ; Q_{1}, Q_{2}, Q_{3} ;$ and $Q_{0}, Q_{1}, Q_{2}$ respectively, i.e., $H_{0}=X$ $\cap\left\{X_{1}=0\right\}, H_{1}=X \cap\left\{X_{0}=0\right\}$ and $H_{2}=X \cap\left\{X_{3}=0\right\}$.

On $X$ there are at least five lines (cf. Figure 2):

$$
\begin{array}{ll}
l_{1}=\overline{Q_{0} Q_{2}}: \quad X_{1}=X_{3}=0 \\
l_{2}=\overline{Q_{0} Q_{3}}: \quad X_{1}=X_{2}=0
\end{array}
$$




$$
\begin{array}{ll}
l_{3}=\overline{Q_{1} Q_{2}}: \quad X_{0}=X_{3}=0 \\
l_{4}=\overline{Q_{1} Q_{3}}: \quad X_{0}=X_{2}=0 \\
l_{5}=\overline{Q_{2} Q_{3}}: \quad X_{0}=X_{1}=0
\end{array}
$$

Let $\tilde{l}_{i}$ denote the proper transform of $l_{i}$ to $\tilde{S}$. Let $X_{\text {smooth }}$ be the smooth part of $X$. Then

Lemma 4.7. $l_{i} \backslash\left\{Q_{0}, \ldots, Q_{3}\right\} \subset X_{\text {smooth }}(1 \leq i \leq 5)$.

Proof. Let $\mathscr{H}_{i}$ be the hyperplane $\left\{X_{i}=0\right\}$ in $\mathbf{P}^{3}$ with homogeneous coordinate $\left(X_{0}: \cdots: \check{X}_{i}: \cdots: X_{3}\right)$. Then we have $X . \mathscr{H}_{3}=l_{1}+l_{3}+C$, where $C$ denote the curve defined in $\mathscr{H}_{3}$ by

$$
X_{0}^{2} X_{1}+X_{0} X_{1}^{2}+X_{0} X_{2}^{2}+c_{1} X_{0} X_{1} X_{2}+c_{4} X_{1} X_{2}^{2}=0
$$

Moreover $C \cap l_{1}=\left\{Q_{0}, Q_{2}\right\}$ and $C \cap l_{3}=\left\{Q_{1}, Q_{2}\right\}$. Therefore $\left(l_{1}+l_{3}\right) \backslash\left\{Q_{0}\right.$, $\left.Q_{1}, Q_{2}\right\} \subset X_{\text {smooth }}$. From the symmetry, we have also $\left(l_{2}+l_{4}\right) \backslash\left\{Q_{0}, Q_{1}, Q_{3}\right\}$ $\subset X_{\text {smooth }}$. Since $X . \mathscr{H}_{0}=2 l_{3}+2 l_{4}+l_{5}, l_{5} \backslash\left\{Q_{2}, Q_{3}\right\} \subset X_{\text {smooth }}$.

Now, let us look into properties near $Q_{0}$ and $Q_{1}$.

Lemma 4.8. (i) $Q_{0}$ and $Q_{1}$ are not rational double points.

(ii) If $Q_{i}(i=0$ or 1$)$ is minimally elliptic, then the fundamental cycle $Z$ of its resolution satisfies $Z^{2}=-2$.

Proof. It suffices to prove for $Q_{0}$. Let $\tilde{\sigma}: \tilde{\mathbf{P}} \rightarrow \mathbf{P}^{3}$ be the blowing-up at $Q_{0}$, $\mathbf{E}=\tilde{\sigma}^{-1}\left(Q_{0}\right), X^{\prime}$ the proper transform of $X$ and $\sigma: X^{\prime} \rightarrow X$ the restriction of $\tilde{\sigma}$ to $X^{\prime}$. Set $U=\left\{X_{0} \neq 0\right\}$, and $x=X_{1} / X_{0}, y=X_{2} / X_{0}, z=X_{3} / X_{0}$, so that $(x, y, z)$ form coordinates of $U$ with $Q_{0}=(0,0,0)$. Then

$$
X \cap U=\left\{\begin{array}{c}
x^{2}+x^{3}+x y^{2}+x z^{2}+c_{1} x^{2} y+c_{2} x^{2} z+c_{3} x y z+c_{4} x^{2} y^{2} \\
+c_{5} x^{2} z^{2}+c_{6} y^{2} z^{2}+c_{7} x^{2} y z+c_{8} x y^{2} z+c_{9} x y z^{2}+c_{10} x y^{2} z^{2}=0
\end{array}\right\} .
$$

$\tilde{U}:=\tilde{\sigma}^{-1}(U)$ is covered by three coordinate neighbourhoods $\tilde{U}_{x}, \tilde{U}_{y}$ and $\tilde{U}_{z}$. Let us consider $\tilde{U}_{z}$ with coordinates $u=x / z, v=y / z, w=z$. On $\tilde{U}_{z}, \mathbf{E}$ is defined by $w=0$, and $X^{\prime}$ by

$$
u^{2}+u^{3} w+u v^{2} w+u w+c_{1} u^{2} v w+c_{2} u^{2} w+c_{3} u v w+c_{4} u^{2} v^{2} w^{2}
$$




$$
+c_{5} u^{2} w^{2}+c_{6} v^{2} w^{2}+c_{7} u^{2} v w^{2}+c_{8} u v^{2} w^{2}+c_{9} u v w^{2}+c_{10} u v^{2} w^{3}=0 .
$$

Hence $\{u=w=0\}=X^{\prime} \cap \mathbb{E} \cap \tilde{U}_{z}$ is the one-dimensional singular locus of $X^{\prime} \cap \tilde{U}_{z}$. By the symmetry, we conclude that $X^{\prime} \cap \mathbf{E}=\sigma^{-1}\left(Q_{0}\right)$ is the singular locus of $X^{\prime}$ over $Q_{0}$. The blowing-up of a rational double point or a minimally elliptic singularity such that the fundamental cycle $Z$ of its resolution satisfies $Z^{2}=-1$ has only isolated singularity (cf. Brieskorn [B], Laufer [L]).

Corollary 4.9. (i) $p_{g}(\tilde{S})=0$.

(ii) $\operatorname{dim} R^{1} \pi_{*} \mathcal{O}_{\tilde{S}}=4+q(\tilde{S})$.

Proof. By the Lemma and since $Q_{2}$ and $Q_{3}$ are triple points, none of $Q_{0}, \ldots, Q_{3}$ are rational double points. Hence $\pi^{-1}\left(Q_{i}\right) \subset \operatorname{Supp} \tilde{D}(0 \leq i \leq 3)$. But there are no hyperplane which passes through all $Q_{i}$, and so $p_{g}(\tilde{S})=\operatorname{dim} H^{0}\left(\tilde{S}, \mathcal{O}_{\tilde{S}}(\tilde{H}-\tilde{D})\right)=0$. (ii) follows from (i) and the exact sequence

$$
0 \rightarrow H^{1}\left(X, \mathcal{O}_{X}\right) \rightarrow H^{1}\left(\tilde{S}, \mathcal{O}_{\tilde{s}}\right) \rightarrow R^{1} \pi_{*} \mathcal{O}_{\tilde{s}} \rightarrow H^{2}\left(X, \mathcal{O}_{X}\right) \rightarrow H^{2}\left(\tilde{S}, \mathcal{O}_{\tilde{S}}\right)
$$

Lemma 4.10. For $i=0,1$, the multiplicity of any component of $\pi^{-1}\left(Q_{i}\right)$ in $\pi^{*} H_{i}$ is greater than one.

Proof. Again it suffices to prove for $i=0$. We use the notations in the proof of Lemma 4.8. If $\mathscr{H}_{1}$ is the hyperplane defined by $X_{1}=0$, then $\tilde{\sigma}^{*} \mathscr{H}_{1}$ is defined by $u w=0$ on $\tilde{U}_{z}$. And both $\{u=0\}$ and $\{w=0\}$ contain $\sigma^{-1}\left(Q_{0}\right) \cap \tilde{U}_{z}$. Therefore, if $\varphi: \tilde{X}^{\prime} \rightarrow X$ is a resolution of $X$, which factors through $X^{\prime}$, then the multiplicity of any component of $\varphi^{-1}\left(Q_{0}\right)$ in $\varphi^{*} H_{0}$ is greater than 1 . Hence the Lemma follows because any resolution of $X$ factors through $\tilde{S}$.

Next, we examine the blow-up of $X$ at $Q_{2}$ and $Q_{3}$. Let $\pi^{\prime}: \tilde{S}^{\prime} \rightarrow X$ be a resolution of $X$, which factors through the blowing-up $X^{\prime \prime}$ of $X$ at $Q_{2}$ and $Q_{3}$. We assume furthermore that $\tilde{S}^{\prime}$ is the minimal resolution of $X^{\prime \prime}$ if we restrict the morphism $\tilde{S}^{\prime} \rightarrow X^{\prime \prime}$ to the normal part of $X^{\prime \prime}$. Let $Z_{i}^{\prime}$ denote the fundamental cycle of $\pi^{\prime-1}\left(Q_{i}\right)(0 \leq i \leq 3)$, and $\tilde{l}_{i}^{\prime}\left[\right.$ resp. $\left.l_{i}^{\prime}\right]$ the proper transform of $l_{i}$ to $\tilde{S}^{\prime}$ [resp. $\left.X^{\prime \prime}\right](1 \leq i \leq 5)$. Then

Lemma 4.11. $\tilde{S}^{\prime}$ has an elliptic fibration $\tilde{f}: \tilde{S}^{\prime} \rightarrow \mathbb{P}^{1}$ such that 
(i) $\tilde{l}_{i}^{\prime}$ is contained in a fibre of $\tilde{f}(1 \leq i \leq 5)$;

(ii) every multiple fibre of $\tilde{f}$ has multiplicity two;

(iii) the number of multiple fibres of $\tilde{f}$ is at most four;

(iv) $2\left(Z_{0}^{\prime}+\tilde{l}_{1}^{\prime}+\tilde{l}_{2}^{\prime}\right)$ and $2\left(Z_{1}^{\prime}+\tilde{l}_{3}^{\prime}+\tilde{I}_{4}^{\prime}\right)$ are multiple fibres of $\tilde{f}$. Moreover, $Z_{0}^{\prime}$ and $Z_{1}^{\prime}$ are reduced.

Proof. Let $\mathscr{H}_{\alpha} \cong \mathbb{P}^{2}$ be the hyperplane $\left\{X_{1}=\alpha X_{0}\right\}$ in $\mathbb{P}^{3}$ with homogeneous coordinates $\left(X_{0}: X_{2}: X_{3}\right)$. Then $\mathscr{H}_{\alpha}: X$ is of the form $l_{5}+C_{\alpha}$, where $C_{\alpha}$ is a curve of degree 4 on $\mathscr{H}_{\alpha}$ defined by

$$
\begin{aligned}
G_{\alpha}= & \left(\alpha^{2}+\alpha^{3}\right) X_{0}^{4}+\alpha^{2} c_{1} X_{0}^{3} X_{2}+\alpha^{2} c_{2} X_{0}^{3} X_{3} \\
& +\left(\alpha+\alpha^{2} c_{4}\right) X_{0}^{2} X_{2}^{2}+\left(\alpha+\alpha^{2} c_{5}\right) X_{0}^{2} X_{3}^{2}+\left(\alpha c_{3}+\alpha^{2} c_{7}\right) X_{0}^{2} X_{2} X_{3} \\
& +\alpha c_{8} X_{0} X_{2}^{2} X_{3}+\alpha c_{9} X_{0} X_{2} X_{3}^{2}+\left(c_{6}+\alpha c_{10}\right) X_{2}^{2} X_{3}^{2} \\
= & 0,
\end{aligned}
$$

while $l_{5}$ is defined by $X_{0}=0$ on $\mathscr{H}_{\alpha}$. Therefore, if $\alpha$ is general, then $C_{\alpha} \cap l_{5}=\left\{Q_{2}, Q_{3}\right\}$ since $c_{10} \neq 0$. Let $x=X_{0} / X_{3}$ and $y=X_{2} / X_{3}$. Then the defining equation of $C_{\alpha}$ near $Q_{3}$ on $\mathscr{H}_{\alpha}$ is

$$
\begin{aligned}
g_{\alpha} & =\left(\alpha+\alpha^{2} c_{5}\right) x^{2}+\alpha c_{9} x y+\left(c_{6}+\alpha c_{10}\right) y^{2}+(\text { higher terms }) \\
& =0 .
\end{aligned}
$$

From this and the symmetry we see that $C_{\alpha}$ has two nodes at $Q_{2}$ and $Q_{3}$, and that their branches are transversal to $l_{5}$ at $Q_{2}$ and $Q_{3}$, provided that $\alpha$ is general. Therefore the proper transform $\tilde{C}_{\alpha}$ of $C_{\alpha}$ to $\tilde{S}^{\prime}$ is a curve of $p_{a}\left(\tilde{C}_{\alpha}\right)=1$ and is disjoint from $\tilde{l}_{5}$. Moreover $\tilde{C}_{\alpha} \cap \tilde{C}_{\alpha^{\prime}}=\emptyset$ for general $\alpha, \alpha^{\prime}$. Hence we deduce that $\left|\tilde{C}_{\alpha}\right|$ defines a (quasi-)elliptic fibration $\tilde{f}: \tilde{S}^{\prime} \rightarrow \mathbf{P}^{1}$. For $\alpha=\infty$, we set $\mathscr{H}_{\infty}=\left\{X_{0}=0\right\}$ and $\mathscr{H}_{\infty} . X=l_{5}+C_{\infty}$. Fix a general $\alpha_{0} \in \mathbf{P}^{1}$ and let $L=\pi^{\prime *}\left(C_{\alpha_{0}}+l_{5}\right)-\tilde{C}_{\alpha_{0}}-\tilde{l}_{5}^{\prime}$. Then Supp $L=\pi^{\prime-1}\left(Q_{2}\right) \cup \pi^{\prime-1}\left(Q_{3}\right)$ by Lemma 4.7. We set $\tilde{C}_{\alpha}=\pi^{\prime *}\left(C_{\alpha}+l_{5}\right)-L-\tilde{l}_{5}^{\prime}$ for every $\alpha \in \mathbb{P}^{1}$, then $\tilde{C}_{\alpha}$ is a fibre of $\tilde{f}$. If we take $\alpha$ satisfying $c_{6}+\alpha c_{10}=0$, so that $X_{0} \mid G_{\alpha}$, we have $l_{5} \subset C_{\alpha}$ and so $\tilde{l}_{5}^{\prime} \subset \tilde{C}_{\alpha}$. For $\alpha=0$ and $\infty$, we have $C_{0}=2 l_{1}+2 l_{2}$ and $C_{\infty}=2 l_{3}+2 l_{4}$, and so $2 \tilde{l}_{1}^{\prime}+2 \tilde{l}_{2}^{\prime} \subset \tilde{C}_{0}$ and $2 \tilde{l}_{3}^{\prime}+2 \tilde{l}_{4}^{\prime} \subset \tilde{C}_{\infty}$. Hence (i) is proved.

Let $m_{\alpha}$ denote the multiplicity of the fibre $\tilde{C}_{\alpha}$, then $g_{\alpha}=h_{\alpha}^{m_{\alpha}}$ for some $h_{\alpha}$. Suppose $m_{\alpha} \geq 2$. Then the part of degree 2 of $g_{\alpha}$ is a square of a linear form. Therefore $\left(\alpha c_{9}\right)^{2}-4\left(\alpha+\alpha^{2} c_{5}\right)\left(c_{6}+\alpha c_{10}\right)=0$. This equation has at most three solution in $k$, and hence there exist at most four $\alpha \in \mathbf{P}^{1}$ such 
that $m_{\alpha} \geq 2$. This proves (iii).

To prove (ii) and (iv), we look at the morphism $X^{\prime \prime} \rightarrow X$ near $Q_{3}$. Let $\tilde{\tau}: \tilde{\mathbf{P}}^{\prime} \rightarrow \mathbb{P}^{3}$ be the blowing-up at $Q_{2}$ and $Q_{3}, \mathbf{E}^{\prime}=\tilde{\tau}^{-1}\left(Q_{3}\right)$ and $\tau=\tilde{\tau}_{\mid X^{\prime \prime}}: X^{\prime \prime} \rightarrow X$. Set $V=\left\{X_{3} \neq 0\right\}$ and $x=X_{0} / X_{3}, y=X_{1} / X_{3}, z=X_{2} / X_{3}$, so that $(x, y, z)$ are coordinates of $V$ with $Q_{3}=(0,0,0)$. Then

$$
X \cap V=\left\{\begin{array}{c}
x^{3} y^{2}+x^{2} y^{3}+x^{2} y z^{2}+x^{2} y+c_{1} x^{2} y^{2} z \\
+c_{2} x^{2} y^{2}+c_{3} x^{2} y z+c_{4} x y^{2} z^{2}+c_{5} x y^{2} \\
+c_{6} x z^{2}+c_{7} x y^{2} z+c_{8} x y z^{2}+c_{9} x y z+c_{10} y z^{2}=0
\end{array}\right\}
$$

$\tilde{V}:=\tilde{\tau}^{-1}(V)$ is covered by three coordinate neighbourhoods $\tilde{V}_{x}, \tilde{V}_{y}$, and $\tilde{V}_{z}$. Let us consider $\tilde{V}_{x}$ with coordinates $u=x, v=y / x, w=z / x$. On $\tilde{V}_{x}, \mathbb{E}^{\prime}$ is defined by $u=0$ and $X^{\prime \prime}$ by

$$
\begin{gathered}
u^{2} v^{2}+u^{2} v^{3}+u^{2} v w^{2}+v+c_{1} u^{2} v^{2} w+c_{2} u v^{2}+c_{3} u v w+c_{4} u^{2} v w^{2} \\
+c_{5} v^{2}+c_{6} w^{2}+c_{7} u v^{2} w+c_{8} u v w^{2}+c_{9} v w+c_{10} v w^{2}=0 .
\end{gathered}
$$

So we see first that $X^{\prime \prime}$ is non-singular at $Q=(0,0,0)$. Since $l_{2}=\{y=z=0\}$ on $V, l_{2}^{\prime} \cap \tilde{V} \subset \tilde{l}_{2}^{\prime} \cap \tilde{V}_{x}$ and $\tilde{l}_{2}^{\prime}$ is defined by $v=w=0$ on $\tilde{V}_{x}$. On the other hand, $l_{5}^{\prime} \cap \tilde{V}_{x}=\emptyset$ and $l_{1}^{\prime} \cap \tilde{V}_{x}=\emptyset$. Let $E=\mathbb{E}^{\prime} \cdot X^{\prime \prime}$. Then $E$ is non-singular at $Q$. Moreover we have that $\tau^{*}\left(2 l_{1}+2 l_{2}+l_{5}\right)=X^{\prime \prime} . \tilde{\tau}^{*} \mathscr{H}_{0}=2 l_{2}^{\prime}+E$ on $\tilde{V}_{x}$ and that $l_{2}^{\prime}$ and $E$ meet only at $Q$ transversally. From these we have that $\pi^{\prime *}\left(2 l_{1}+2 l_{2}+l_{5}\right)=2 \tilde{l}_{2}^{\prime}+D_{3}^{\prime}$ over $\tilde{V}_{x}$ for some effective divisor $D_{3}^{\prime}$ with Supp $D_{3}^{\prime}=\pi^{\prime-1}\left(Q_{3}\right)$ and $\tilde{l}_{2}^{\prime} D_{3}^{\prime}=1$. Hence $\left(D_{3}^{\prime}-L\right) \tilde{l}_{2}^{\prime}=0$. Since $\tilde{C}_{0}$ is connected, it follows from Lemma 4.7 and the symmetry that $\tilde{C}_{0}=D_{0}^{\prime}+2 \tilde{l}_{1}^{\prime}+2 \tilde{l}_{2}^{\prime}$ for some effective divisor $D_{0}^{\prime}$ such that $\operatorname{Supp} D_{0}^{\prime}=\pi^{\prime-1}\left(Q_{0}\right)$. In particular we have $\widetilde{C}_{0} D_{3}^{\prime}=2$, which implies (ii). On the other hand, $\pi^{\prime *}\left(C_{0}+l_{5}\right)=\pi^{\prime *} H_{0}$. So, by Lemma 4.10, all components of $D_{0}^{\prime}$ has multiplicity greater than one in $D_{0}^{\prime}$. Hence $\tilde{C}_{0}$ is a multiple fibre of multiplicity two. Hence we can write $\tilde{C}_{0}=2 \tilde{F}_{0}$. Let $\mu^{\prime}: \tilde{S}^{\prime} \rightarrow S$ be a successive blowing-downs of $(-1)$-curves in fibres of $\tilde{f}$ such that the induced fibration $f: S \rightarrow \mathbb{P}^{1}$ is relatively minimal. Let $F_{0}=\mu_{*}^{\prime} \widetilde{F}_{0}$ and $\bar{C}_{0}=2 F_{0}$. Then $\bar{C}_{0}$ is a fibre of $f$ and $\tilde{C}_{0}=\mu^{\prime *} \bar{C}_{0}$. Note that $F_{0}$ is reduced. Moreover the induced morphism $D_{0}^{\prime} \rightarrow F_{0}$ is surjective. In fact, since $Q_{0}$ is not a rational singularity (Lemma 4.8 (i)),

$$
\sup _{\substack{D>0 \\ \operatorname{Supp} D \subseteq \pi^{\prime-1}\left(Q_{0}\right)}} p_{a}(D)>0
$$


by Artin [A], and hence the surjectivity follows from Corollary 4.6. In particular $\tilde{l}_{1}^{\prime}$ and $\tilde{l}_{2}^{\prime}$ are exceptional for $\mu^{\prime}$. Since $\pi^{\prime}$ is the minimal resolution with respect to $Q_{0}, \tilde{l}_{1}^{\prime}$ and $\tilde{l}_{2}^{\prime}$ are the only possible $(-1)$-curves in $\tilde{C}_{0}$, and their multiplicities in $\widetilde{F}_{0}$ is equal to one. This implies that $\widetilde{F}_{0}$ is reduced. Since $\tilde{F}_{0} \Gamma=0$ for any irreducible component $\Gamma$ of $\widetilde{F}_{0}$, we see that $\widetilde{F}_{0}-\tilde{l}_{1}^{\prime}-\tilde{l}_{2}^{\prime}$ coincides with the fundamental cycle $Z_{0}^{\prime}$. Therefore, with the symmetry, (iv) and the last part of the Lemma is proved.

Let $\mu^{\prime}: \widetilde{S}^{\prime} \rightarrow S$ be, as in the proof of Lemma 4.11, successive blowing-downs of $(-1)$-curves in fibres of $\tilde{f}$ so that we get a relatively minimal (quasi-)elliptic fibration $f: S \rightarrow \mathbf{P}^{1}$.

We consider in two cases.

Case 1: $q(S)=0$. Lemma 4.8 (i) and Corollary 4.9 (ii) say that $Q_{0}, Q_{1}, Q_{2}, Q_{3}$ are all minimally elliptic singularities. By Laufer [L] and Lemma 4.8 (ii), the minimal resolution factors the blowing-up of $Q_{0}, \ldots, Q_{3}$. Hence $\tilde{S}^{\prime}=\tilde{S}$, and so we write $\mu=\mu^{\prime}: \tilde{S} \rightarrow S$. For every $i, \tilde{l}_{i}$ lies in a fibre of $\tilde{f}\left(\right.$ Lemma 4.11 (i)) and $\tilde{l}_{i} \cong \mathbf{P}^{1}$. Hence $\tilde{l}_{i}^{2}<0$. Moreover $\tilde{H} \tilde{l}_{i}=1$ and $\tilde{D} \tilde{l}_{i} \geq 2$ because $l_{i}$ passes through two points from $\left\{Q_{0}, \ldots, Q_{3}\right\}$. So, by $\tilde{H} \tilde{l}_{i}-\tilde{D} \tilde{l}_{i}+\tilde{l}_{i}^{2}$ $=-2$, we have $\tilde{D} \tilde{l}_{i}=2$ and $\tilde{l}_{i}^{2}=-1$. Hence $\tilde{l}_{i}$ is contracted to a point by $\mu$. Let $\tilde{D}=\widetilde{E}_{0}+\cdots+\widetilde{E}_{3}$, where $\tilde{E}_{i}$ is the part of $\tilde{D}$ such that $\operatorname{Supp} \widetilde{E}_{i}=\pi^{-1}\left(Q_{i}\right)$. In our case $\tilde{E}_{i}$ coincides with the fundamental cycle of $\pi^{-1}\left(Q_{i}\right)$ (Laufer [L]). Therefore, from Lemma 4.7 and 4.11 (and its proof),

$$
\begin{aligned}
& \pi^{*} H_{0}=2 \tilde{E}_{0}+\tilde{E}_{2}+\tilde{E}_{3}+2 \tilde{l}_{1}+2 \tilde{l}_{2}+\tilde{l}_{5}+\Delta_{0} \\
& \pi^{*} H_{1}=2 \tilde{E}_{1}+\tilde{E}_{2}+\tilde{E}_{3}+2 \tilde{l}_{3}+2 \tilde{l}_{4}+\tilde{l}_{5}+\Delta_{1},
\end{aligned}
$$

where $\Delta_{0}$ and $\Delta_{1}$ are effective divisors whose support is included in $\operatorname{Supp}\left(\tilde{E}_{2}+\tilde{E}_{3}\right)$. On the other hand, since $\tilde{D} \tilde{l}_{i}=2$, we have

$$
\begin{array}{ll}
\tilde{E}_{0} \tilde{l}_{1}=\tilde{E}_{2} \tilde{l}_{1}=1, & \tilde{E}_{1} \tilde{l}_{1}=\tilde{E}_{3} \tilde{l}_{1}=0, \\
\tilde{E}_{0} \tilde{l}_{2}=\tilde{E}_{3} \tilde{l}_{2}=1, & \tilde{E}_{1} \tilde{l}_{2}=\tilde{E}_{2} \tilde{l}_{2}=0, \\
\tilde{E}_{1} \tilde{l}_{3}=\tilde{E}_{2} \tilde{l}_{3}=1, & \tilde{E}_{0} \tilde{l}_{3}=\tilde{E}_{3} \tilde{l}_{3}=0, \\
\tilde{E}_{1} \tilde{l}_{4}=\tilde{E}_{3} \tilde{l}_{4}=1, & \tilde{E}_{0} \tilde{l}_{4}=\tilde{E}_{2} \tilde{l}_{4}=0, \\
\tilde{E}_{2} \tilde{l}_{5}=\tilde{E}_{3} \tilde{l}_{5}=1, & \tilde{E}_{0} \tilde{l}_{5}=\tilde{E}_{1} \tilde{l}_{5}=0 .
\end{array}
$$

Moreover, from Lemma 4.8 and Laufer [L], $\widetilde{E}_{0}^{2}=\widetilde{E}_{1}^{2}=-2, \widetilde{E}_{2}^{2}=\widetilde{E}_{3}^{2}=-3$. Hence we can calculate 


$$
\Delta_{0}^{2}=\left\{\pi^{*} H_{0}-\left(2 \tilde{E}_{0}+\tilde{E}_{2}+\tilde{E}_{3}+2 \tilde{l}_{1}+2 \tilde{l}_{2}+\tilde{l}_{5}\right)\right\}^{2}=0
$$

which means $\Delta_{0}=0$. Similarly $\Delta_{1}=0$. Therefore we obtain

$$
\begin{aligned}
2 K_{\tilde{\mathbf{S}}} & \sim \pi^{*} H_{0}+\pi^{*} H_{1}-2\left(\tilde{E}_{0}+\tilde{E}_{1}+\tilde{E}_{2}+\tilde{E}_{3}\right) \\
& =2\left(\tilde{l}_{1}+\tilde{l}_{2}+\tilde{l}_{3}+\tilde{l}_{4}+\tilde{l}_{5}\right) .
\end{aligned}
$$

This implies that the contraction of $\tilde{l}_{1}, \ldots, \tilde{l}_{5}$ coincides with $S$ and that $S$ is a minimal surface with $2 K_{S} \sim 0$. Together with $p_{g}(S)=0$ (Corollary 4.9 (i)) and $q(S)=0$, we conclude that $S$ is an Enriques surface. Let $E_{i}=\mu_{*} \tilde{E}_{i}(0 \leq i \leq 3)$. By Lemma 4.11 (iv), $E_{0}$ and $E_{1}$ are the halfpencils of the elliptic fibration $f$. Let us show that $E_{2}$ and $E_{3}$ are also halfpencils. By (***) we see $E_{i}^{2}=0$ and $p_{a}\left(\tilde{E}_{i}\right)=p_{a}\left(E_{i}\right)=1$ for every $i$. But $\left|E_{i}\right|$ does not define an elliptic fibration since $E_{i} E_{j}=1$ for some $j$. Since $Q_{0}, Q_{1}, Q_{2} \in H_{2}$ and $Q_{3} \notin H_{2}$, we have that $\pi^{*} H_{2}=\widetilde{E}_{0}+\widetilde{E}_{1}+\widetilde{E}_{2}+\widetilde{E}_{3}^{\prime}$ for some effective divisor $\widetilde{E}_{3}^{\prime}$, which is disjoint from $\tilde{E}_{3}$. Hence

$$
K_{\tilde{S}} \sim \pi * H_{2}-\tilde{D}=\tilde{E}_{3}^{\prime}-\tilde{E}_{3}
$$

and so $K_{S} \sim E_{3}^{\prime}-E_{3}$, where $E_{3}^{\prime}=\mu_{*} \tilde{E}_{3}^{\prime} . \quad E_{3}$ and $E_{3}^{\prime}$ have no common components and $2 E_{3}-2 E_{3}^{\prime} \sim 2 K_{S} \sim 0$. Thus $2 E_{3}$ defines an elliptic fibration, and so $E_{3}$ is a halfpencil. Also $E_{2}$ is a halfpencil. Moreover (***) implies that $E_{0} E_{2}=E_{2} E_{3}=E_{3} E_{0}=1, E_{0}, E_{1}, E_{2}, E_{3}$ meet at five different points, which we call $P_{1}, \ldots, P_{5}$ (cf. Figure 1 with $E_{1}$ and $E_{1}^{\prime}$ replaced by $E_{0}$ and $E_{1}$ respectively), and that $\mu$ is the blowing-up at $P_{1}, \ldots, P_{5}$. Moreover

$$
\begin{aligned}
\mu_{*} \tilde{H} & \sim \mu_{*}\left(\pi^{*} H_{0}\right)=2 E_{0}+E_{2}+E_{3} \\
& \sim E_{0}+E_{1}+E_{2}+E_{3}+K_{S}, \\
\tilde{H} & \sim \pi^{*} H_{0}=\mu^{*}\left(\mu_{*}\left(\pi^{*} H_{0}\right)\right)-\tilde{l}_{1}-\cdots-\tilde{l}_{5} \\
& \sim \mu^{*}\left(\mu_{*} \tilde{H}\right)-\tilde{l}_{1}-\cdots-\tilde{l}_{5} .
\end{aligned}
$$

Therefore $X$ can be reconstructed from $S$ in the way described in $\S 3$.

Case 2: $q(S)>0$. From the canonical bundle formula and Lemma 4.11, we have $K_{S} \sim f^{*} \mathscr{M}+\Sigma_{i=0}^{m} F_{i}$, where $\mathscr{M}$ is a line bundle on $\mathbb{P}^{1}$ of degree $\chi\left(\mathcal{O}_{S}\right)-2$ and $\left\{2 F_{0}, \ldots, 2 F_{m}\right\}$ is the set of the multiple fibres of $f$ with $1 \leq m \leq 3$. Let $F$ be a general fibre of $f$. Then $K_{S} \equiv\left(\chi\left(0_{S}\right)-2+1 / 2(m+1)\right) F$.

Obviously $\kappa(S) \leq 1$. If $\kappa(S)=1$, then $\chi\left(\mathcal{O}_{S}\right)-2+1 / 2(m+1)>0$, and hence $\chi\left(\mathcal{O}_{S}\right)>0$. This is impossible since $q(S)>0$ and $p_{g}(S)=0$ (Corollary 4.9 (i)). If 
$\kappa(S)=0$, then $S$ is a (quasi-)hyperelliptic surface. But if $\operatorname{ch}(k)=0$, then no normal quintic surface can be birationally a hyperelliptic surface (NakamuraUmezu $[\mathrm{N}-\mathrm{U}]$ ). We will show that no (quasi-)hyperelliptic surface appears in our situation in any characteristic. Assume that $S$ is a (quasi-)hyperelliptic surface. Then a similar argument as in the proof of Proposition 1.1-3 shows that $\operatorname{dim} R^{1} \pi_{*} \mathcal{O}_{\tilde{S}}=5$ and that the natural map $\mu: \tilde{S} \rightarrow S$ consists of at most five blow-ups. Moreover any rational curve $C$ on $S$ is, if it exists, a cuspidal curve with $C^{2}=0$ (Bombieri-Mumford [B-M]). From these we can deduce that the map $\mu$ is just the contraction of the disjoint $(-1)$-curves $\tilde{l}_{1}, \ldots, \tilde{l}_{5}$ and that there exists a non-singular rational curve $\Gamma$ in $\tilde{D}$ which is not exceptional for $\mu$. Then we have $\Gamma \tilde{l}_{i} \geq 2$ for some $i$. Since $\tilde{l}_{i}$ meets at least two components of $\tilde{D}$, we obtain $K_{\tilde{S}} \tilde{l}_{i}=(\tilde{H}-\tilde{D}) \tilde{l}_{i} \leq-2$, and hence a contradiction.

Suppose $\kappa(S)=-\infty$. Then $S$ is birationally a ruled surface over a curve of genus $q(S)$. Since there are curves of arithmetic genus 1 on $S$, we obtain $q(S)=1, \chi\left(\mathcal{O}_{S}\right)=0$ and $m=1$ or 2. Moreover $K_{S}^{2}=0$ implies that $S$ itself is minimal. Let $g: S \rightarrow E$ ( $E$ is an elliptic curve) denote the ruling of $S$. Since all fibres of $f$ are mapped surjectively onto $E$, they are non-singular elliptic curves. Corollary 4.9 (ii) with our assumption says that there exists a unique $i_{0} \quad\left(0 \leq i_{0} \leq 3\right)$ such that $Q_{i_{0}}$ is a singularity of geometric genus 2 (i.e. $\operatorname{dim}\left(R^{1} \pi_{*} \mathcal{O}_{\tilde{S}}\right)_{Q_{1_{0}}}=2$ by the definition due to Wagreich [W]), and other $Q_{i}$ 's are minimally elliptic singularities. We may assume $i_{0}=0$ or 3 . If $i_{0}=0$, then $\tilde{S}^{\prime}=\tilde{S}$. Let us show that the morphism $\mu^{\prime}: \tilde{S}^{\prime} \rightarrow S$ factors through $\tilde{S}$ if $i_{0}=3$, too. Assume to the contrary. Let $\tilde{S}^{\prime}=\tilde{S}_{0} \stackrel{v_{1}}{\rightarrow} \tilde{S}_{1} \stackrel{v_{2}}{\rightarrow} \cdots \stackrel{v_{h}}{\rightarrow} \tilde{S}_{h}=\tilde{S}$ be a sequence of blowing-downs from $\tilde{S}^{\prime}$ to $\tilde{S}$. Let $A_{i} \subset \tilde{S}_{i-1}$ denote the exceptional (-1)-curve for $v_{i}$. The proper transform $\tilde{A}_{i}$ of $A_{i}$ to $\tilde{S}^{\prime}$ is a component of $\pi^{\prime-1}\left(Q_{3}\right)$. Then there exists $h_{0}\left(0 \leq h_{0} \leq h\right)$ such that $\mu^{\prime}$ factors through $\tilde{S}_{h_{0}}$ but does not through $\tilde{S}_{h_{0}+1}$. That is, $\tilde{A}_{1}, \ldots, \tilde{A}_{h_{0}}$ are contained in fibres of $\tilde{f}$ but $\tilde{A}_{h_{0}+1}$ is not. Let $\rho: \tilde{S}_{h_{0}} \rightarrow S$ denote the induced morphism. Since $\rho_{*} A_{h_{0}+1}$ is a rational curve, it is a fibre of $g$. On the other hand, $\mu_{*}^{\prime}\left(Z_{i}^{\prime}\right)(i=0,1)$ is not contained in a fibre of $g$ because of Corollary 4.6 and $p_{a}\left(Z_{i}^{\prime}\right)=1$. Hence $\left(\rho_{*} A_{h_{0}+1}\right)\left(\mu_{*}^{\prime} Z_{i}^{\prime}\right)>0$ for $i=0,1$. Since $A_{h_{0}+1}$ is disjoint from $\left(v_{h_{0}} \circ \cdots \circ v_{1}\right)_{*}\left(Z_{i}^{\prime}\right)$ $(i=0,1)$ and since $\mu_{*}^{\prime}\left(Z_{0}^{\prime}\right) \cap \mu_{*}^{\prime}\left(Z_{1}^{\prime}\right)=\emptyset$ by Lemma 4.11 (iv), there lie on $\rho_{*} A_{h_{0}+1}$ at least two centers of blowing-ups in $\rho$. Therefore we get $A_{h_{0}+1}^{2} \leq-2$. This is a contradiction, and hence we conclude that $\mu^{\prime}: \tilde{S}^{\prime} \rightarrow S$ factors through $\tilde{S}$. Let $\mu: \tilde{S} \rightarrow S$ be the induced morphism. Then, as in case 1 , we see that $\tilde{l}_{1}, \ldots, \tilde{l}_{5}$ are disjoint $(-1)$-curves and are exceptional for $\mu$, and that $\tilde{D} \tilde{l}_{i}=2$ for every $i$. Let $\tilde{D}=\tilde{D}_{0}+\cdots+\tilde{D}_{3}$ such that $\operatorname{Supp} \tilde{D}_{i}=\pi^{-1}\left(Q_{i}\right)$. Set $D_{i}=\mu_{*} \tilde{D}_{i}$. 
Since $Q_{i}$ is not a rational singularity, $D_{i}$ is not contained in a fibre of $g$ (Artin [A] and Corollary 4.6), and hence $D_{i}$ has a component $E_{i}$ such that $g\left(E_{i}\right) \geq 1$. By Hidaka-Watanabe $[\mathrm{H}-\mathrm{W}], Q_{i_{0}}$ is also an elliptic singularity. By definition (Wagreich [W]),

$$
\sup _{\substack{D>0 \\ \operatorname{Supp} D \subseteq \pi^{\prime}-1}} p_{a}(D)=1 .
$$

Let $\widetilde{E}_{i}$ denote the proper transform of $E_{i}$ on $\tilde{S}$. Then $\widetilde{E}_{i}$ is a non-singular elliptic curve $(0 \leq i \leq 3), \tilde{D}_{i}=\tilde{E}_{i}$ for $i \neq i_{0}$, and

$$
\tilde{D}_{i_{0}}=\gamma \tilde{E}_{i_{0}}+(\text { trees of non-singular rational curves }) \text {. }
$$

Hence $\pi: \tilde{S} \rightarrow X$ is also the minimal good resolution of singularities of $X$, and we can apply results on elliptic sequence. What we need here is the following (Yau [Y1], [Y2], Tomari [T]): There exists a decomposition $\tilde{D}_{i_{0}}=Z_{i_{0} .0}+Z_{i_{0}, 1}$ $+\cdots+Z_{i_{0} . l}+\tilde{E}_{i_{0}}(l \geq 0)$ such that

(i) $Z_{i_{0} .0}$ is the fundamental cycle of $\pi^{-1}\left(Q_{i_{0}}\right)$;

(ii) $Z_{i_{0}, 0}>Z_{i_{0}, 1}>\cdots>Z_{i_{0}, l}>\tilde{E}_{i_{0}}$;

(iii) $Z_{i_{0.0}}^{2} \leq Z_{i_{0} .1}^{2} \leq \cdots \leq Z_{i_{0}, l}^{2} \leq \tilde{E}_{i_{0}}^{2}$;

(iv) $\tilde{D}_{i_{0}}^{2}=\Sigma_{j=0}^{l} Z_{i_{0}, j}^{2}+\tilde{E}_{i_{0}}^{2}$;

(v) mult $_{Q_{i_{0}}} X \geq-\Sigma_{j=0}^{l} Z_{i_{0}, j}^{2}$;

(vi) $\tilde{E}_{i_{0}} Z_{i_{0} .0}=0$.

Assume $i_{0}=0$. By Lemma 4.11 we have that $2 E_{0}$ is a fibre of $f$ and that $\mu^{*} E_{0}=Z_{0.0}+\tilde{l}_{1}+\tilde{l}_{2}$, which is reduced. Moreover we have seen that $\tilde{l}_{1}$ and $\tilde{l}_{2}$ are $(-1)$-curves and are disjoint from each other. Hence we get $Z_{0.0}^{2}=-2$. Since $Q_{0}$ is a double point, we have $l=0$ and so $\tilde{D}_{0}=Z_{0,0}+\widetilde{E}_{0}$. Therefore

$$
\mathcal{O}_{\widetilde{E}_{0}} \cong \mathcal{C}_{\widetilde{E}_{0}}\left(K_{\widetilde{E}_{0}}\right) \cong \mathcal{O}_{\widetilde{E}_{0}}\left(\tilde{H}-\tilde{D}+\widetilde{E}_{0}\right) \cong \mathcal{O}_{\widetilde{E}_{0}}\left(-Z_{0,0}\right)
$$

and hence $\mathcal{O}_{\widetilde{E}_{0}}\left(\tilde{E}_{0}\right) \cong \mathcal{O}_{\tilde{E}_{0}}\left(-\left(Z_{0,0}-\widetilde{E}_{0}\right)\right)$. This happens only if every exceptional curve of $\mu$, such that its center of blowing-up lies on $E_{0}$ or its proper transform, is a component of $Z_{0,0}$, and $\mathcal{O}_{E_{0}}\left(E_{0}\right) \cong \mathcal{O}_{E_{0}}$. But this is impossible since $\mathcal{O}_{E_{0}}\left(E_{0}\right)$ is of order 2 because $2 E_{0}$ is a multiple fibre.

In what follows we assume $i_{0}=3$. Then $\tilde{D}_{i}=\tilde{E}_{i}$ for $0 \leq i \leq 2$, and 
$\tilde{E}_{0}^{2}=\tilde{E}_{1}^{2}=-2, \tilde{E}_{2}^{2}=-3$ (Laufer [L] and Lemma 4.8). By Lemma 4.11, the morphism $\mu$ is over $E_{0}$ [resp. over $E_{1}$ ] blowing-ups at two different points, say $P_{1}$ and $P_{2}$ [resp. $P_{3}$ and $\left.P_{4}\right]$, and $\mu^{-1}\left(P_{j}\right)=\tilde{l}_{j}(1 \leq j \leq 4)$. Hence $E_{0}$ intersects $D_{3}$ transversally only at $P_{2}$, and $E_{0}$ and $D_{3}$ are non-singular at $P_{2}$. Therefore $E_{0} D_{3}=1$. Let $G$ be a general fibre and $C_{0}$ a minimal section of $g$. Put $e=-C_{0}^{2}$. Then $e \geq-1$ by Nagata [N]. From

$$
-2 C_{0}-e G \equiv K_{S} \equiv(-2+1 / 2(m+1)) F,
$$

we have $0 \leq C_{0}\left(-K_{S}\right)=-e$, and so $e=0$ or -1 .

Let us first suppose $e=0$. Then $C_{0}$ is contained in a fibre of $f$. Since $C_{0} G=1$ and $f$ has no section, $2 C_{0}$ is a multiple fibre. By Lemma 4.11 (iv), there is another multiple fibre $2 C_{1}$. Then there are no multiple fibres of $f$ other than $2 C_{0}$ and $2 C_{1}$ since $f$ induces a double cover of rational curves $G \rightarrow \mathbf{P}^{1}$. We may assume $C_{0}=E_{0}$ and $C_{1}=E_{1}$. Hence $C_{0} D_{3}=E_{0} D_{3}=1$. If $D_{3}$ has no rational comoponents, then $D_{3}=\gamma E_{3}$ with $\gamma \geq 2$, which contradicts with $C_{0} D_{3}=1$. Hence $D_{3}$ contains a fibre $G_{0}$ of $g$ as a component. Since $C_{0}\left(D_{3}-G_{0}\right)=0$, we conclude that $D_{3}=\gamma E_{3}+G_{0}$ with $\gamma \geq 2$ and $E_{3}$ is a (non-multiple) fibre of $f$. Since the restriction of $g$ to $E_{3}$ is an unramified morphism of degree 2, $E_{3}$ and $G_{0}$ intersect transversally at two different points $R_{1}$ and $R_{2}$. Let $\tilde{G}_{0}$ denote the proper transform of $G_{0}$ to $\tilde{S}$ and $\tilde{R}_{i}$ the point on $\tilde{E}_{3}$ over $R_{i}$ for $i=1,2$. We may assume that the connected component of $\tilde{D}_{3}-\gamma \widetilde{E}_{3}$ which contains $\widetilde{G}_{0}$ meets $\tilde{E}_{3}$ at $\tilde{R}_{1}$ and that $R_{2}$ is one of the centers of the blowing-ups in $\mu$. Let $n$ denote the number of blowing-ups in $\mu$. Then

$$
\begin{aligned}
n & =K_{S}^{2}-K_{\tilde{S}}^{2}=-(\tilde{H}-\tilde{D})^{2}=-\left(5+\tilde{D}_{0}^{2}+\cdots+\tilde{D}_{3}^{2}\right) \\
& =2-\tilde{D}_{3}^{2}=2-\tilde{E}_{3}^{2}-\sum_{j=0}^{l} Z_{3, j}^{2} .
\end{aligned}
$$

Since $Q_{3}$ is a triple point, we have $-\Sigma_{j=0}^{l} Z_{3, j}^{2} \leq 3$, therefore $n \leq 5-\tilde{E}_{3}^{2}$. This implies that the centers of the blowing-ups in $\mu$, other than $P_{1}, \ldots, P_{4}$, lie on $E_{3}$ or its proper transform except for at most one point. Since $\tilde{D} \tilde{I}_{5}=2$, $\tilde{D}_{3} \tilde{l}_{5}=\tilde{E}_{2} \tilde{l}_{5}=1$. Therefore $\tilde{l}_{5}$ meets exactly one component of $\tilde{D}_{3}$, whose multiplicity in $\tilde{D}_{3}$ is equal to one. This component is not $\widetilde{E}_{3}$ since $\gamma \geq 2$. Let $P$ be the point obtained by contracting $\tilde{l}_{5}$. Then $P$ is the center of a blowing-up in $\mu$, other than $P_{1}, \ldots, P_{4}$, which does not lie on a proper transform of $E_{3}$. From this and $\tilde{E}_{3}^{2} \geq Z_{3,0}^{2} \geq-3$, and since $\tilde{D}_{3}$ has no $(-1)$-curve as a 
component, we can deduce that the configuration of $\tilde{D}_{3}$ is

$$
\tilde{E}_{3}+(\text { less than three) chains of non-singular rational curves, }
$$

and that the fundamental cycle $Z_{3,0}$ is reduced. By $\tilde{E}_{3} Z_{3,0}=0$, we have that $-\widetilde{E}_{3}^{2}$ is equal to the number of the chains of rational curves in $\tilde{D}_{3}$, which is less than three. Furthermore we note that $\mathcal{O}_{E_{3}}\left(E_{3}\right) \simeq \mathcal{O}_{E_{3}}$. If $\widetilde{E}_{3}^{2}=-1$, then we obtain $Z_{3,0}=\widetilde{E}_{3}+\widetilde{G}_{0}$ and $\widetilde{E}_{3} \widetilde{G}_{0}=\widetilde{R}_{1}$, and so $l=0, \tilde{D}_{3}=2 \widetilde{E}_{3}+\widetilde{G}_{0}$. Therefore

$$
\mathcal{O}_{\tilde{E}_{3}} \cong \mathcal{O}_{\tilde{E}_{3}}\left(K_{\tilde{E}_{3}}\right) \cong \mathcal{O}_{\tilde{E}_{3}}\left(\tilde{H}-\tilde{D}+\widetilde{E}_{3}\right) \cong \mathcal{O}_{\tilde{E}_{3}}\left(-\widetilde{E}_{3}-\tilde{G}_{0}\right) \cong \mathcal{O}_{\tilde{E}_{3}}\left(\tilde{R}_{2}-\tilde{R}_{1}\right)
$$

and hence $R_{1}=R_{2}$, which is a contradiction. Assume $\tilde{E}_{3}^{2}=-2$. Since $Z_{3,0}^{2} \leq \cdots \leq Z_{3, l}^{2} \leq \tilde{E}_{3}^{2}=-2$ and $-\Sigma_{j=0}^{l} Z_{3, j}^{2} \leq 3$, we have $l=0$ and $\tilde{D}_{3}=\widetilde{E}_{3}+Z_{3,0}$. Hence $\mathcal{O}_{\tilde{E}_{3}} \cong \mathcal{O}_{\tilde{E}_{3}}\left(-Z_{3,0}\right)$ as above, and so $\mathcal{O}_{\tilde{E}_{3}}\left(Z_{3,0}-\widetilde{E}_{3}\right) \cong \mathcal{O}_{\tilde{E}_{3}}\left(-\widetilde{E}_{3}\right)$. Let $\widetilde{R}$ be the point on $\tilde{E}_{3}$ such that $\left(Z_{3.0}-\tilde{E}_{3}\right) \widetilde{E}_{3}=\widetilde{R}_{1}+\tilde{R}$. We note that $\widetilde{R} \neq \tilde{R}_{1}$. Moreover let $R^{\prime}$ be the other center than $R_{2}$ of the blowing-ups in $\mu$, which lies on the proper transform of $E_{3}$. Then we have $\mathcal{O}_{\widetilde{E}_{3}}\left(\widetilde{R}_{1}+\widetilde{R}\right)$ $\cong \mathcal{O}_{\tilde{E}_{3}}\left(\widetilde{R}_{2}+\widetilde{R}^{\prime}\right)$, where $\widetilde{R}^{\prime}$ denotes the point on $\widetilde{E}_{3}$ over $R^{\prime}$. If $\widetilde{R}=\widetilde{R}_{2}$, then $\widetilde{R}^{\prime}=\widetilde{R}_{1}$, which is impossible because then the exceptional curve of the blowing-up at either $R_{1}$ or $R_{2}$, which should be a component of $Z_{3,0}$, remains (-1)-curve on $\tilde{S}$. But $\tilde{R} \neq \widetilde{R}_{2}$ implies $\widetilde{R}=\widetilde{R}^{\prime}$, and so $R_{1}=R_{2}$, again a contradiction.

Suppose $e=-1$. Then $(2-1 / 2(m+1)) C_{0} F=C_{0}\left(-K_{S}\right)=1$, and hence $m=2$ and $C_{0} F=2$ since $f$ has no section. Let $s$ and $t$ be the integers such that $E_{0} \equiv s C_{0}+t G$. Then we obtain $s+t=1$ by $C_{0} E_{0}=1 / 2 C_{0} F=1$, and $s+2 t=0$ by $E_{0}^{2}=0$ and $s=E_{0} G>0$. Therefore $s=2$ and $t=-1: E_{0} \equiv 2 C_{0}-G$. Hence, from $E_{0} D_{3}=1$, we have

$$
E_{0}\left(D_{3}-G\right)=-1<0 \text {. }
$$

This implies that $D_{3}$ has no rational curve as a component. Hence $D_{3}=\gamma E_{3}$, which contradicts $E_{0} D_{3}=1$ since $\gamma \geq 2$.

Therefore the case of $\kappa=-\infty$ does not occur, and we have completed the proof of Theorem 4.3.

Remark. In the proof above, we use the assumption on the singularity of $X$ to exclude the case that $\kappa=-\infty$ and that $X$ has five simple elliptic singularities. In fact, we can construct normal quintic surfaces $X$ from elliptic ruled surfaces $S$ with $e=-1$ or 0 (in the latter case $S=\mathbb{P}\left(\mathcal{O}_{E} \oplus \mathscr{L}\right)$, where $\mathscr{L}$ is an invertible sheaf on the elliptic curve $E$ such that $\mathscr{L} \not \mathcal{O}$ and $\mathscr{L}^{\otimes 2} \cong \mathcal{O}_{E}$ ), such that $X$ have five simple elliptic singularities at $Q_{0}(1: 0: 0: 0), Q_{1}(0: 1: 0: 0)$, 
$\mathrm{Q}_{2}(0: 0: 1: 0), Q_{3}(0: 0: 0: 1)$, and another point $Q$ and satisfy Lemma 4.11 . But the author does not know if their defining equations can be reduced to the form $(* *)$.

\section{Appendix}

Here we assume that the ground field $k$ is an algebraically closed field of arbitrary characteristic.

Proposition. Let $E_{1}$ and $E_{2}$ be effective divisors on a non-singular surface $S$ such that:

(i) $E_{1}$ and $E_{2}$ have no (-1)-curve as a component;

(ii) $\left|m_{1} E_{1}\right|$ and $\left|m_{2} E_{2}\right|$ define respective elliptic fibrations for some $m_{1}, m_{2} \geq 1$;

(iii) $E_{1} E_{2}=1$.

Then $E_{1}$ and $E_{2}$ have no common components.

Proof. Assumption (ii) implies $E_{i} C=0$ for every irreducible component $C$ of $E_{i}$. Moreover, with (i), any curve, whose support is properly contained in $\operatorname{Supp} E_{i}$, can be contracted to rational double points.

Set $E_{1}=Z_{1}+F_{1}$ and $E_{2}=Z_{2}+F_{2}$, where $\operatorname{Supp} Z_{1}=\operatorname{Supp} Z_{2}, F_{1}, F_{2}, Z_{1}$ have no common components, and $Z_{1}, Z_{2}, F_{1}, F_{2}$ are all effective. Put $A=\left(Z_{1}\right)_{\text {red }}=\left(Z_{2}\right)_{\text {red }}$. Assumimg $A \neq 0$, we shall deduce a contradiction. Assumption (iii) implies $F_{1} \neq 0$ and $F_{2} \neq 0$. Hence $A$ is the (possibly disconnected) exceptional set of the minimal resolution of rational double points. Let $Z_{0}$ denote the fundamental cycle of any connected component of $A$. It is clear that $Z_{1} C, Z_{2} C \leq 0$ for every irreducible component $C$ of $A$, and so we obtain $Z_{0} \leq Z_{1}, Z_{2}$. Therefore we have

$$
Z_{1} Z_{2} \leq Z_{1} Z_{0} \leq Z_{0}^{2}=-2 \text {. }
$$

On the other hand, we have from (iii)

$$
1=E_{1} E_{2}=\left(Z_{1}+F_{1}\right) E_{2}=F_{1} E_{2}=F_{1} Z_{2}+F_{1} F_{2} .
$$

Here $F_{1} Z_{2} \geq 1$ since $E_{1}$ is connected, and $F_{1} F_{2} \geq 0$. Therefore $F_{1} Z_{2}=1$. But this contradicts $Z_{1} Z_{2} \leq-2$, because

$$
0=E_{1} Z_{2}=Z_{1} Z_{2}+F_{1} Z_{2}
$$




\section{References}

[A] Artin, M., On isolated rational singularities of surfaces, Amer. J. Math., 88 (1966), 129-136.

[B] Brieskorn, E., Über die Auflösung gewisser Singularitäten von holomorphen Abbildungen, Math. Ann., 166 (1966), 76-102.

[B-M] Bombieri, E. and Mumford, D., Enriques' classification of surfaces in char. p, II, In: W. L. Baily, Jr. and T. Shioda (eds.): Complex analysis and algebraic geometry (pp23-42) Iwanami-Shoten, Tokyo, 1977.

[B-P] Barth, W. and Peters, C., Automorphisms of Enriques surfaces, Invent. Math., 73 (1983), 383-411.

[B-P-V] Barth, W., Peters, C. and Van de Ven, A., Compact complex surfaces (Ergeb. Math. Grenzgeb., 3. Folge, Bd 4), Springer, Berlin Heidelberg New York Tokyo, 1984.

[Ca] Castelnuovo, G., Sulle superficie di genere zero, Mem. delle Soc. Ital. delle Scienze, ser. III, 10 (1895).

[Co] Cossec, F., On the Picard group of Enriques surfaces, Math. Ann., 271 (1985), 577-600.

[Co-D1] Cossec, F. and Dolgachev, I. V., Smooth rational curves on Enriques surfaces, Math. Ann., 272 (1985), 369-384.

[Co-D2] _ Enriques Surfaces I(Progr. Math. 76), Birkhäuser, Boston Basel Berlin, 1989.

[H-W] Hidaka, F. and Watanabe, K., Normal Gorenstein surfaces with ample anti-canonical divisor, Tokyo J. Math., 4 (1981), 319-330.

[Ki] Kim, Y., On normal quintic Enriques surfaces, Thesis, Univ. of Michigan (1991).

[Ko] Kondō, S., The rationality of the moduli space of Enriques surfaces, Comp. Math., 91 (1994), 159-173.

[L] Laufer, H., On minimally elliptic singularities, Amer. J. Math., 99 (1977), 1257-1295.

[N] Nagata, M., On self-intersection number of a section on a ruled surface, Nagoya Math.J., 7 (1970), 191-196.

[N-U] Nakamura, I. and Umezu, Y., Nonexistence of normal quintic abelian surfaces in $\mathbf{P}^{3}$, Tokyo J. Math., 18 (1995), 369-382.

[S] Stagnaro, E., Constructing Enriques surfaces from quintics in $P_{K}^{3}$, In: C. Ciliberto et al. (eds.): Algebraic geometry-open problems Ravello 1982 (Lect. Notes Math., 997, pp400-403), Springer, Berlin Heidelberg New York Tokyo, 1983.

[T] Tomari, M., A $p_{g}$-formula and elliptic singularities, Publ. RIMS, Kyoto Univ., 21 (1985), 297-354.

[U] Umezu, Y., On birational models of Enriques surfaces in $\mathbb{P}^{3}$, Proc. Japan Acad., 70 (1994), 137-139.

[W] Wagreich, P., Elliptic singularities of surfaces, Amer. J. Math., 92 (1970), 419-454.

[Y1] Yau, S. S. -T., Hypersurface weighted dual graphs of normal singularities of surfaces, Amer. J. Math., 101 (1979), 761-812.

[Y2] - Gorenstein singularities with geometric genus equal to two, Amer. J. Math., 101 (1979), 813-854. 